\title{
UNIQUENESS FOR SOLUTIONS OF FOKKER-PLANCK EQUATIONS RELATED TO SINGULAR SPDE DRIVEN BY LÉVY AND CYLINDRICAL WIENER NOISE
}

\author{
SVEN WIESINGER
}

\begin{abstract}
We generalize recent results concerning uniqueness of solutions to Fokker-Planck equations (FPE) related to singular Hilbert space-valued SPDE from the (cylindrical) Wiener noise case to the case of SPDE driven by noise with jumps. Using a different space of test functions, we can relax the usual integrability assumptions and obtain more general uniqueness results for FPE, even in the case of SPDE driven by Wiener noise.
\end{abstract}

\section{INTRODUCTION}

Recently, in a series of papers (see e.g. [BDPR09, [BDPR11]), the uniqueness of solutions to Fokker-Planck equations (FPE) related to Kolmogorov operators, which are the generators for classes of singular SPDE, has been established for the case of SPDE driven by (cylindrical) Wiener noise. The aim of this paper is, to provide a detailed study of the analogous problem for SPDE driven by noise with jumps. Our main tools include a newly established test function space (see Subsection 1.5 below), which seems to be particularly well-suited for our analysis (cf. Remarks 2.4 and 2.5), and some new results for the space-time generator of the related linear SPDE, which are also proved in this paper. We focus on the case of SPDE with a merely measurable nonlinear drift part $F$. Let us note, however, that similar uniqueness results can be achieved for FPE associated to SPDE with m-dissipative drift; we refer to Remark 2.6 below for more details.

With respect to the length of this paper, we concentrate here only on the question of uniqueness of solutions for FPE. Nevertheless, we briefly address the existence problem in Remark 6.2 below.

1.1. Our Framework. We consider the equation

$$
\left\{\begin{aligned}
\mathrm{d} X(t) & =[A X(t)+F(t, X(t))] \mathrm{d} t+\mathrm{d} Y(t) \\
X(s) & =x \in H, \quad 0 \leq s \leq t \leq T,
\end{aligned}\right.
$$

where the self-adjoint operator $A$ is the infinitesimal generator of a $C_{0}$-semigroup of operators on a separable real Hilbert space $H$ denoted by $\left(e^{t A}\right)$, which we assume to be quasi-contractive; $F: D(F) \subset H \rightarrow H$ a measurable map; and $T$ a finite positive real number. We consider $Y$ as the sum of a centered Lévy process $J$ in

2000 Mathematics Subject Classification. Primary 60H15; Secondary 35R60.

The author would like to thank Michael Röckner for some very helpful discussions. Financial support of the German Science Foundation (DFG) through CRC701 and IRTG1132 is gratefully acknowledged. 
$H$ with characteristic triplet $[b, 0, M]$ and a (possibly cylindrical) Wiener process $\sqrt{Q} W$. We identify $H$ with its dual $H^{*}$ via the Riesz isomorphism.

Recall, that for a process $Y$ of this type the characteristic function takes the form $\mathbb{E}\left[e^{i\langle\xi, Y(t)\rangle}\right]=e^{-t \lambda(\xi)}, \xi \in H$, where the so-called characteristic exponent $\lambda: H \rightarrow \mathbb{C}$ of $Y$ can be represented in the following Lévy-Khintchine decomposition:

$$
\lambda(\xi)=-i\langle\xi, b\rangle+\frac{1}{2}\langle\xi, Q \xi\rangle-\int_{H} \exp [i\langle\xi, x\rangle]-1-\frac{i\langle\xi, x\rangle}{1+|x|^{2}} M(\mathrm{~d} x) .
$$

The so-called characteristic triplet $[b, Q, M]$ of $b \in H, Q \in L(H)$ and the measure $M$ uniquely characterizes the process $Y$. If $Y$ is a Lévy process, then $Q$ is nonnegative, symmetric and trace-class. However, to include the case of cylindrical Wiener processes in our framework, we do not assume the trace-class property for $Q$. The measure $M$ in this characterization is a Lévy measure; that is, a Borel measure satisfying $M(\{0\})=0$ and $\int_{H}\left(1 \wedge|x|^{2}\right) M(\mathrm{~d} x)<\infty$.

If $Y$ is a Lévy process, then $\xi \mapsto \lambda(\xi)$ is negative definitive, Sazonov-continuous and we have $\lambda(0)=0$. Due to our relaxed condition on $Q$, the second summand on the right-hand side of (1.1) is no longer Sazonov-continuous. Note, that the two other summands, considered separately, still have this property (see e.g. Par67, Ch. VI, Thms. 2.4 and 4.8]).

It is well-known, that for any $C_{0}$-semigroup $\left(e^{t A}\right)$ the stochastic convolution $Y_{A}(t):=\int_{0}^{t} e^{s A} \mathrm{~d} Y(s), t \geq 0$, is well-defined; see e.g. CM87, PZ07. We denote its distribution for $t \in[0, T]$ by $\mu_{t}$; the Fourier transform takes the form

$$
\hat{\mu}_{t}(\xi)=\exp [-\underbrace{\int_{0}^{t} \lambda\left(e^{s A} \xi\right) \mathrm{d} s}_{=: \lambda_{t}(\xi)}] \quad \text { for all } t \geq 0, \xi \in H,
$$

where, as shown in FR00, $\lambda_{t}$ can be decomposed as

$$
\lambda_{t}(\xi)=-i\left\langle\xi, b_{t}\right\rangle+\frac{1}{2}\left\langle\xi, Q_{t} \xi\right\rangle-\int_{H} \exp [i\langle\xi, x\rangle]-1-\frac{i\langle\xi, x\rangle}{1-|x|^{2}} M_{t}(\mathrm{~d} x) .
$$

As shown in the same reference, the characteristic triplet $\left[b_{t}, Q_{t}, M_{t}\right]$ of $Y_{A}$ can be expressed in terms of $\left(e^{t A}\right)$ and the characteristic triplet $[b, Q, M]$ of $Y$. If $Q$ is of trace-class, then $Q_{t}$ inherits this property. Similarly, if $M$ is a Lévy measure on $H$, then so is $M_{t}$, and if $b \in H$ then $b_{t} \in H$.

The transition semigroup $\left(S_{t}\right)$ related to (SPDE) in the case of $F \equiv 0$ (linear case) takes the form

$$
S_{t} \varphi(x):=\mathbb{E}[\varphi(X(t, 0, x))]=\int_{H} \varphi\left(e^{t A} x+y\right) \mu_{t}(\mathrm{~d} y), \quad \varphi \in \mathcal{B}_{b}(H) .
$$

It belongs to the family of so-called generalized Mehler semigroups, which have been introduced in BRS96 and studied e.g. in [FR00, [SS01], LR02], App07] (see also the references therein). In LR02, the restriction of the infinitesimal generator $U$ of $\left(S_{t}\right)$ to a suitable space of test functions $\mathcal{W}_{A}$ (see Subsection 1.5 below) has been identified as

$$
U \psi(x)=\int_{H}[i\langle A \xi, x\rangle-\lambda(\xi)] \cdot e^{i\langle\xi, x\rangle} \mathcal{F}^{-1}(\psi(\cdot))(\mathrm{d} \xi), \quad \psi \in \mathcal{W}_{A},
$$

where $\mathcal{F}^{-1}$ denotes the inverse Fourier transform. As pointed out in LR02, p. 300], we have for all $\psi \in \mathcal{W}_{A}$, that $S_{t} \psi(x) \stackrel{t \rightarrow 0}{\longrightarrow} \psi(x)$ for all $x \in H$. The fact, that this convergence can be established only pointwise in $H$ (and not with respect to 
the supremum norm in the function space), takes $\left(S_{t}\right)$ out of reach of the theory of $C_{0}$-semigroups. Instead, we use the theory of so-called $\pi$-semigroups (see e.g. Cer95], Pri99, Man06 and the references therein) in our analysis.

1.2. The Fokker-Planck equation. To discuss our approach in the semilinear case, let us assume for a moment, that $F$ is sufficiently regular to establish existence and uniqueness of a pathwise solution to (SPDE) (cf. e.g. [PZ07]). We denote the resulting family of transition evolution operators as $\left(P_{s, t}\right)_{0 \leq s \leq t \leq T}$. The restriction $L_{0}$ of its infinitesimal generator to suitable test functions $\psi \in \mathcal{W}_{T, A}$ can be specified as

$$
L_{0} \psi(t, \cdot)=D_{t} \psi(t, \cdot)+\langle D \psi(t, \cdot), F(t, \cdot)\rangle+U \psi(t, \cdot), \quad \psi \in \mathcal{W}_{T, A}, t \in[s, T],
$$

and the family of probability measures $\eta=\left(\eta_{t}\right)_{t \in[s, T]}$ on $H$ defined by $\eta_{t}(\mathrm{~d} x):=$ $\left(P_{s, t}^{*} \zeta(\mathrm{d} x)\right.$ ) (where $P^{*}$ denotes the adjoint of the operator $P$, and $\zeta$ is a probability measure on $H$ ) is known to fulfill the Fokker-Planck equation

$$
\int_{H} \psi(t, x) \eta_{t}(\mathrm{~d} x)=\int_{H} \psi(s, x) \zeta(\mathrm{d} x)+\int_{s}^{t} \int_{H} L_{0} \psi(r, x) \eta_{r}(\mathrm{~d} x) \mathrm{d} r
$$

for all $\psi \in \mathcal{W}_{T, A}$ and almost all $t \in[s, T]$. In our hypotheses below, we introduce conditions to make sure, that the integrals in (FPE) exist.

At the heart of the approach followed in this article lies the observation, that it is possible to identify (by approximation) the Kolmogorov operator $L$ even for equations of type (SPDE) with singular coefficients, for which a pathwise solution does not necessarily exist. In this case, the aim is to find a family $\eta$, which solves (FPE), and thus understand the development of the distribution of the solution to (SPDE). Recent references on the study of existence and uniqueness of solutions to Fokker-Planck equations in finite dimensions include e.g. BDPR08, [Fig08, [LBL08, [RZ10] and the references therein. (See also related fundamental work on transport equations in DL89.) In more recent years, for the first time the focus of attention has been extended also to the infinite-dimensional case; see e.g. AF09, BDPR09], BDPR10, BDPR11] and the references therein. However, to the best of our knowledge, all of the current and past research on this approach via FPE seems to have focused exclusively on the case of SPDE perturbed by Wiener noise. The aim of this paper is, to establish uniqueness for equations of type (FPE) in the case of a singular drift coefficient $F$ and noise with jumps.

1.3. Structure of this paper. In the remaining part of the current section, we introduce our technical framework, in particular the test function space $\mathcal{W}_{T, A}$ (cf. Subsection 1.5 below). In Section 2, we specify the hypotheses underlying our approach and present the main result. A major part of the work in this paper, which provides the basis for the main uniqueness proof, is the generalization of results for the linear case (i.e. $F=0$ ) from the Wiener noise case to the case of noise with jumps. This is done in Section 3 , where we extend results from the theory of generalized Mehler semigroups to the case of space- and time-dependent function spaces. Section 4 contains some results for the case of a regular drift coefficient $F$, which are needed in the approximation procedure within the main uniqueness proof. On these foundations, we carry out the proof of our main uniqueness result in Section 5. In Section 6, we discuss a class of examples of time-dependent reactiondiffusion type. 
1.4. Spaces of functions and measures, which are used below. We denote the inner product in $H$ by $\langle\cdot, \cdot\rangle$ and the norm by $|\cdot|$. As usual, $L(H)$ denotes the space of bounded linear operators on $H$, and $\|\cdot\|_{L(H)}$ the operator norm.

We denote the Borel $\sigma$-algebra on $H$ as $\mathcal{B}(H)$ and the Banach space of bounded, $\mathcal{B}(H)$-measurable functions $H \rightarrow \mathbb{R}$ as $\mathcal{B}_{b}(H)$, with supremum norm $\|\cdot\|_{0}$.

By $\mathcal{C}_{u}(H)$, we denote the closed subspace of $\mathcal{B}_{b}(H)$ of all functions $H \rightarrow \mathbb{R}$, which are uniformly continuous; the space of continuous elements of $\mathcal{B}_{b}(H)$ is denoted by $\mathcal{C}_{b}(H)$. The space $\mathcal{C}_{u, k}(H), k \in \mathbb{N}$, contains all functions $\varphi: H \rightarrow \mathbb{R}$, such that $x \mapsto \frac{\varphi(x)}{1+|x|^{k}}$ is in $\mathcal{C}_{u}(H)$. We use the norm

$$
\|\varphi\|_{u, k}:=\sup _{x \in H} \frac{|\varphi(x)|}{1+|x|^{k}} \quad \text { for } k \in \mathbb{N}, \varphi \in \mathcal{C}_{u, k}(H) .
$$

For any $k \in \mathbb{N}$, the space $\mathcal{C}_{u}^{k}(H)$, is made up of all functions in $\mathcal{C}_{u}(H)$ with continuous and bounded derivatives of order $\ell$ for any $\ell \leq k$.

By $\mathcal{C}\left([0, T] ; \mathcal{C}_{u}(H)\right)$, we denote the space of all functions $\varphi:[0, T] \times H \rightarrow \mathbb{R}$, such that $x \mapsto \varphi(t, x)$ is in $\mathcal{C}_{u}(H)$ for any $t \in[0, T]$, and $t \mapsto \varphi(t, \cdot)$ is continuous with respect to the sup-norm on $\mathcal{C}_{u}(H)$. On $\mathcal{C}\left([0, T] ; \mathcal{C}_{u}(H)\right)$, we define the norm

$$
\|\varphi\|_{0, T}:=\sup _{t \in[0, T]}\|\varphi(t, \cdot)\|_{0} .
$$

Furthermore, $\mathcal{C}\left([0, T] ; \mathcal{C}_{u, k}(H)\right), k \in \mathbb{N}$, is the space of all functions $\varphi:[0, T] \times H \rightarrow$ $\mathbb{R}$, such that the mapping $(t, x) \mapsto \frac{\varphi(t, x)}{1+|x|^{k}}$ is in $\mathcal{C}\left([0, T] ; \mathcal{C}_{u}(H)\right)$. We use the norm

$$
\|\varphi\|_{u, k, T}:=\sup _{t \in[0, T]}\|\varphi(t, \cdot)\|_{u, k} \quad \text { for } k \in \mathbb{N}, \varphi \in \mathcal{C}\left([0, T] ; \mathcal{C}_{u, k}(H)\right) .
$$

The (Frechet) derivative of a function with respect to space is denoted by $D$; the derivative with respect to time is denoted by $D_{t}$.

The Schwartz function space $\mathcal{S}\left(\mathbb{R}^{d} ; \mathbb{C}\right)$ is the space of all functions $\varphi: \mathbb{R}^{d} \rightarrow \mathbb{C}$, which are differentiable infinitely often and fulfill

$$
\|\varphi\|_{\alpha, \beta}:=\sup _{x \in \mathbb{R}^{d}}\left|x^{\alpha} D^{\beta} \varphi(x)\right|<\infty
$$

for all $d$-tuples $\alpha, \beta$ of nonnegative integers.

The space of probability measures on $H$ is denoted by $\mathcal{M}_{1}(H)$ and the space of complex-valued measures on $H$ with bounded total variation by $\mathcal{M}_{b}^{\mathbb{C}}(H)$. The Fourier transform of a measure $\mu$ is denoted by $\mathcal{F} \mu=\hat{\mu}$, and the inverse Fourier transform by $\mathcal{F}^{-1}$.

1.5. The test function space. In contrast to recent work on (FPE) in the Wiener noise case, we need a different test function space, which can be understood as a space of (linear combinations of) Fourier transforms of measures in $\mathcal{M}_{b}^{\mathbb{C}}(H)$. The time-independent test function space $\mathcal{W}_{A}$ is used in the literature on generalized Mehler semigroups (see e.g. [BRS96, [LR02]). However, since in our case the coefficients in (SPDE) depend explicitly on time, we introduce a time-dependent version, which we denote by $\mathcal{W}_{T, A}$.

We consider the following spaces of functions:

$\mathcal{W}_{A, \mathbb{C}}:$ Functions $\varphi: H \rightarrow \mathbb{C}$, such that there exists an $m \in \mathbb{N}$ with

$$
\varphi(x)=f_{m}\left(\left\langle\xi_{1}, x\right\rangle, \ldots,\left\langle\xi_{m}, x\right\rangle\right) \text { for all } x \in H,
$$


where $f_{m} \in \mathcal{S}\left(\mathbb{R}^{m} ; \mathbb{C}\right)$ and $\left\{\xi_{i}\right\}_{i \in \mathbb{N}}$ is an orthonormal basis (ONB) of $H$, with each $\xi_{i}$ being an eigenvector of $A$ (see Hypothesis (H.1) below).

$\mathcal{W}_{A}$ : Real-valued elements of $\mathcal{W}_{A, \mathbb{C}}$.

We will sometimes write $\varphi(x)=f_{m}\left(P_{m} x\right)$ for $\varphi \in \mathcal{W}_{A}, x \in H$, where $P_{n}, n \in \mathbb{N}$, denotes the orthogonal projection of $H$ onto $\operatorname{span}\left(\left\{\xi_{1}, \ldots, \xi_{n}\right\}\right)\left(\equiv \mathbb{R}^{n}\right)$, defined by

$$
P_{n} x:=\sum_{j=1}^{n}\left\langle x, \xi_{j}\right\rangle \xi_{j} \quad \text { for all } x \in H .
$$

$\mathcal{W}_{T, A}:$ The linear span of all functions $\psi:[0, T] \times H \rightarrow \mathbb{R}$, such that there is an $m \in \mathbb{N}$ with

$$
\psi(t, x)=\phi(t) \cdot f_{m}\left(\left\langle\xi_{1}, x\right\rangle, \ldots,\left\langle\xi_{m}, x\right\rangle\right) \quad \forall(t, x) \in[0, T] \times H,
$$

where $f_{m} \in \mathcal{S}\left(\mathbb{R}^{m} ; \mathbb{R}\right)$, the $\left\{\xi_{i}\right\}_{i \in \mathbb{N}}$ are chosen as above and $\phi \in \mathcal{C}^{2}([0, T])$, with the additional requirement that $\phi(T)=\phi^{\prime}(T)=\phi^{\prime \prime}(T)=0$.

The following remark relates to the time-independent case in [LR02, Rem. 1.1].

Remark 1.1. Choose any $\psi \in \mathcal{W}_{T, A}$ of the form (1.3). Denote the inverse Fourier transform of $f_{m}$ by $g_{m}: \mathbb{R}^{m} \rightarrow \mathbb{C}$. Note that $g_{m} \in \mathcal{S}\left(\mathbb{R}^{m} ; \mathbb{C}\right)$, see e.g. RS80, Ch. $\mathrm{IX}]$, and that $g_{m}$ is uniquely determined by the requirement, that

$$
f_{m}(y)=\int_{\mathbb{R}^{m}} e^{i(r, y)_{\mathbb{R}^{m}}} g_{m}(r) \mathrm{d} r \quad \text { for all } y \in \mathbb{R}^{m} .
$$

We set

$$
\nu_{m}(\mathrm{~d} r):=g_{m}(r) \mathrm{d} r \quad \text { for } r \in \mathbb{R}^{m} .
$$

Observe that $\nu_{m}$ is in $\mathcal{M}_{b}^{\mathbb{C}}\left(\mathbb{R}^{m}\right)$ for each $m \in \mathbb{N}$. Now consider the embedding

$$
\begin{aligned}
\Pi_{m}: \mathbb{R}^{m} & \rightarrow{ }^{m} \\
\left(r_{1}, \ldots, r_{m}\right) & \mapsto \sum_{j=1}^{m} r_{j} \xi_{j}
\end{aligned}
$$

and define

$$
\nu_{t}:=\phi(t) \nu_{m} \circ \Pi_{m}^{-1} \quad\left(\in \mathcal{M}_{b}^{\mathbb{C}}(H) \text { for any } t \in[0, T]\right) .
$$

Similarly to BLR99, Lem. 1.3] we see, that for all $t \in[0, T]$ and $x \in H$

$$
\begin{gathered}
\mathcal{F}\left(\nu_{t}\right)(x)=\int_{H} e^{i\langle y, x\rangle} \nu_{t}(\mathrm{~d} y)=\phi(t) \cdot \int_{\mathbb{R}^{m}} \exp \left[i \sum_{j=1}^{m} r_{j} \cdot\left\langle\xi_{j}, x\right\rangle\right] g_{m}(r) \mathrm{d} r \\
=\phi(t) \cdot f_{m}\left(\left\langle\xi_{1}, x\right\rangle, \ldots,\left\langle\xi_{m}, x\right\rangle\right)=\psi(t, x) .
\end{gathered}
$$

To motivate the significance of the test function space, we state the following lemma, which we really only need in the case $k=1$.

Lemma 1.2. For any $\varphi \in \mathcal{C}\left([0, T] ; \mathcal{C}_{u, k}(H)\right), k \in \mathbb{N}$, there exists a triple-index sequence $\left\{\psi_{n_{1}, n_{2}, n_{3}}\right\} \subset \mathcal{W}_{T, A}$, such that for all $(t, x) \in[0, T) \times H$

(i) $\left|\psi_{n_{1}, n_{2}, n_{3}}(t, x)\right| \leq\left(\|\varphi\|_{u, k, T}+1\right) \cdot\left(1+|x|^{k}\right) \quad$ for all $n_{1}, n_{2}, n_{3} \in \mathbb{N}$

(ii) $\lim _{n_{1} \rightarrow \infty} \lim _{n_{2} \rightarrow \infty} \lim _{n_{3} \rightarrow \infty} \psi_{n_{1}, n_{2}, n_{3}}(t, x)=\varphi(t, x)$.

The lemma follows from a Stone-Weierstraß argument extending on the basic idea of the proof of DP04, Prop. 1.2]. 
1.6. Spaces of probability kernels. Finally, let us introduce the following notations for spaces of probability kernels, including the solution space for (FPE) denoted by $\mathcal{K}_{s, \zeta}^{0}$. Let $s \in[0, T]$ and $\zeta \in \mathcal{M}_{1}(H)$. We set:

$\mathcal{K}_{s}^{0}$ : positive Borel measures $\eta$ on $[s, T] \times H$, such that $\eta(\mathrm{d} t, \mathrm{~d} x)=\eta_{t}(\mathrm{~d} x) \mathrm{d} t$, where $\eta_{t} \in \mathcal{M}_{1}(H)$ for all $t \in[s, T]$, and $t \mapsto \eta_{t}(B)$ is measurable on $[s, T]$ for all $B \in \mathcal{B}(H)$

$\mathcal{K}_{s, \zeta}^{0}$ : elements $\eta$ of $\mathcal{K}_{s}^{0}$, which fulfill FPE with initial condition $\zeta \in \mathcal{M}_{1}(H)$

$\mathcal{K}_{s, \leq \beta}^{0}$ : elements $\eta$ of $\mathcal{K}_{s}^{0}$, such that there exists a $\beta \geq 0$ with

$$
\int_{[s, T] \times H} L_{0} \psi(r, x) \eta(\mathrm{d} r, \mathrm{~d} x) \leq \beta \cdot \int_{[s, T] \times H} \psi(r, x) \eta(\mathrm{d} r, \mathrm{~d} x)
$$

for all $\psi \in \mathcal{W}_{T, A}$ with $\psi \geq 0$.

Remark 1.3. $\mathcal{K}_{s, \zeta}^{0} \subset \mathcal{K}_{s, \leq \beta}^{0}$ (see e.g. 4.3 below).

\section{Hypotheses AND MAIN RESUlts}

2.1. The linear case. We extend existing results about the generalized Mehler semigroup related to (SPDE) to the case of explicitly time-dependent test functions. These results will be needed later on. Proofs are included in Section 3 We use the following hypotheses:

(H.1) $H$ has an orthonormal basis $\left\{\xi_{i}\right\}_{i \in \mathbb{N}}$ of eigenvectors of $A$, and $A$ is selfadjoint and such that $\langle A x, x\rangle \leq \omega \cdot|x|^{2}$ for some $\omega \geq 0$ and all $x \in D(A)$.

(H.2) The characteristic exponent $\lambda: H \rightarrow \mathbb{C}$ of $Y$ is negative definite and of the form 1.1. We require the trace-class property only for $Q_{t}$, but not for $Q$. The Lévy measure $M$ has finite $q$-th moments for a $q>2$.

For any $n \in \mathbb{N}$ and $F_{n}:=\operatorname{span}\left\{\xi_{1}, \ldots, \xi_{n}\right\}$, the restriction $\left.\lambda\right|_{F_{n}}$ is in $\mathcal{C}^{\infty}\left(F_{n}\right)$. Furthermore, $\operatorname{ker} Q_{t}=\{0\}$ for all $t \geq 0$.

(H.3) $e^{t A}(H) \subset Q_{t}^{1 / 2}(H)$ for all $t>0$.

Furthermore, for each $t \in(0, T]$ there is a $\Lambda_{t} \in L(H)$, such that $Q_{t}^{1 / 2} \Lambda_{t}=e^{t A}$ and $\int_{0}^{T}\left\|\Lambda_{t}\right\|_{L(H)} \mathrm{d} t<\infty$.

Remark 2.1. Hypothesis (H.1) is crucial for the construction of $\mathcal{W}_{T, A}$.

We denote the eigenvalues of $A=A^{*}$ (which due to the self-adjointness are real numbers) by $\alpha_{i}, i \in \mathbb{N}: A \xi_{i}=\alpha_{i} \xi_{i}$ for all $i \in \mathbb{N}$.

Remark 2.2. Note that the assumptions on $Q_{t}$ are standard assumptions for the existence of the stochastic convolution in the Wiener noise case (which is a special case of our situation; cf. e.g. [DPZ92, Hai09]). Some further observations concerning Hypothesis (H.2):

(i) The trace-class property of $Q_{t}$ implies, that $\lambda_{t}$ is Sazonov-continuous. Note, that $\lambda_{t}$ inherits from $\lambda$ the property of being negative definite.

(ii) By construction, the family $\left(\mu_{t}\right)$ of probability measures is infinitely divisible. It can be decomposed as $\mu_{t}=e_{G}\left(M_{t}\right) * N_{Q_{t}} * \delta_{b_{t}}$ for any $t \in[0, T]$, where $e_{G}\left(M_{t}\right)$ denotes the generalized exponent of $M_{t}, N_{Q_{t}}$ the Gaussian measure with covariance operator $Q_{t}$ and $\delta_{b_{t}}$ the Dirac measure with mass in $b_{t}$ (see e.g. [Lin86, Sect. 5.7] for details).

(iii) Clearly, $\int_{H}|x|^{2} M(\mathrm{~d} x)=\int_{\{|x| \leq 1\}}|x|^{2} M(\mathrm{~d} x)+\int_{\{|x|>1\}}|x|^{2} M(\mathrm{~d} x)$. The first summand is finite by virtue of $M$ being a Lévy measure. The second summand is (up to a constant) smaller than the q-th moment of $M$ for 
any $q>2$. Consequently, $M$ has finite second moments. By Lin86, Rem. 5.4.14] this implies, that $\mu_{t}$ also has finite second moments.

(iv) The smoothness condition on finitely based restrictions of $\lambda$ is needed to achieve, that $S_{t}\left(\mathcal{W}_{A}\right) \subset \mathcal{W}_{A}$ (cf. Remark 3.5), which in turn is crucial for the proof of Theorem 1 .

See [LR02, Sect. 3] for a possible approach to the situation without this restriction (however, only in the time-independent case).

Infinite differentiability of finitely-based restrictions of $\lambda$ holds for example, if the Lévy measure $M$ in the Lévy-Khintchine decomposition of $\lambda$ fulfills

$$
M(\mathrm{~d} x)=\mathbb{I}_{\{a \mid \varepsilon \leq\|a\| \leq 1 / \varepsilon\}}(x) \cdot M(\mathrm{~d} x)
$$

for some $\varepsilon>0$ (cf. LR02, Prop. 3.3]).

An example for a negative definite, Sazonov continuous function $\lambda$ : $H \rightarrow \mathbb{R}$, which is $\mathcal{C}^{\infty}$ on $H$ (not only on finitely based restrictions) is

$$
\lambda(\xi)=\frac{m \cdot\|C \xi\|^{2}}{m+\|C \xi\|^{2}}, \quad m>0
$$

where $C: H \rightarrow H$ is assumed to be symmetric, positive definite and of trace-class (cf. [LR02, Rem. 4.2]).

Remark 2.3. For the definition of $B^{1 / 2}$ for a nonnegative operator $B$, see e.g. RS80, Thm. VI.9].

Hypothesis (H.3) is needed for the proof of the integration by parts formula in Lemma 3.1. The latter in turn is required to establish, that the generalized Mehler semigroup $\left(S_{t}\right)$ has the strong Feller property (see Lemma 3.2), on which our proof of the approximation result presented in Theorem 11 below relies.

Consider the space-time homogenization $\left(S_{\tau}^{T}\right)_{\tau \geq 0}$ of the generalized Mehler semigroup $\left(S_{t}\right)$, defined for elements $\varphi$ of

as

$$
\mathcal{C}_{T}\left([0, T] ; \mathcal{C}_{u, 1}(H)\right):=\left\{\varphi \in \mathcal{C}\left([0, T] ; \mathcal{C}_{u, 1}(H)\right) \mid \varphi(T, x)=0 \text { for all } x \in H\right\}
$$

$$
\left(S_{\tau}^{T} \varphi\right)(t, x):= \begin{cases}S_{\tau} \varphi(t+\tau, \cdot)(x) & \text { if } t+\tau \leq T \\ 0 & \text { else. }\end{cases}
$$

Then, the generator $(V, D(V))$ of $\left(S_{\tau}^{T}\right)_{\tau \geq 0}$ on $D(V) \subset \mathcal{C}_{T}\left([0, T] ; \mathcal{C}_{u, 1}(H)\right)$ in the sense of $\pi$-semigroups (similar to $[$ Pri99] $)$ is an extension of

$$
V_{0}:=D_{t}+U, \quad D\left(V_{0}\right):=\mathcal{W}_{T, A}
$$

and we obtain the following approximation result (cf. Corollary 3.12):

Theorem 1. Let $u \in D(V)$ and $\eta$ a finite nonnegative Borel measure on $[0, T] \times H$. Assume, that Hypotheses (H.1) (H.3) hold.

Then, there exist a sequence $\left(\psi_{n}\right)_{n \in \mathbb{N}} \subset \mathcal{W}_{T, A}$ and an $n_{0} \in \mathbb{N}$, such that for a finite $C>0$

$$
\left|\psi_{n}(t, x)\right|+\left|D \psi_{n}(t, x)\right|+\left|V_{0} \psi_{n}(t, x)\right| \leq C \cdot\left(\|V u\|_{u, 1, T}+1\right) \cdot(1+|x|)
$$

for all $(t, x) \in[0, T] \times H, n \geq n_{0}$, and

$$
\psi_{n} \rightarrow u, \quad\left\langle D \psi_{n}, h\right\rangle \rightarrow\langle D u, h\rangle, \quad V_{0} \psi_{n} \rightarrow V u
$$

converge in measure $\eta$ as $n \rightarrow \infty$ for any $h \in H$. 
Remark 2.4. This result has been shown in the Wiener noise case in BDPR09, Cor. A.3] (which in turn generalizes [DPT01, Sect. 2]). However, because of the different family of test functions used in these references, the upper bound achieved there grows as $\left(1+|x|^{2}\right)$ in space. This is essentially due to the fact, that the results equivalent to Lemma 3.10 in these references include continuity of the map $(s, t) \mapsto S_{t} \psi(s, \cdot)$ in the topology of $\mathcal{C}_{u, 2}(H)$ only.

2.2. The semilinear case. We generalize results, which have been achieved for the Wiener noise case in BDPR11, Section 4], to our framework. Proofs are included in Section 5 ,

In addition to Hypotheses (H.1) (H.3), we require the following throughout this part of our work:

(H.4) $F: D(F) \rightarrow H$ is a measurable map, where $D(F) \in \mathcal{B}([0, T] \times H)$.

(As a rule, we set $|F(t, x)|=+\infty$ if $(t, x) \notin D(F)$.)

(H.5) $Q^{-1} \in L(H)$.

Let $s \in[0, T]$ and $\zeta \in \mathcal{M}_{1}(H)$. Set

$\mathcal{K}_{s, \zeta}^{\text {meas }}:=\left\{\left.\eta \in \mathcal{K}_{s, \zeta}^{0}\left|\int_{[s, T] \times H}\right| x\right|^{2}+|F(t, x)|^{2}+|x|^{2} \cdot|F(t, x)|^{2} \eta(\mathrm{d} t, \mathrm{~d} x)<\infty\right\}$.

We obtain the following result (cf. Proposition 5.5):

Theorem 2 (Main Result). Assume that Hypotheses (H.1) (H.5) hold. Then, $\mathcal{K}_{s, \zeta}^{\text {meas }}$ contains at most one element.

Remark 2.5. This result generalizes [BDPR11, Theorem 4.1] from the cylindrical Wiener noise case to the case of (SPDE) driven by the sum of Lévy noise with jumps and a cylindrical Wiener process. The main ingredients in the proof are a gradient estimate for the square-field operator $\Gamma$, which we introduce in Remark 4.4 (ii) below, and the results achieved in Section 3 .

In addition to the generalization, we obtain relaxed moment conditions for $\eta$; in BDPR11, the uniqueness of the solution to (FPE) is shown only in

$$
\left\{\left.\eta \in \mathcal{K}_{s, \zeta}^{0}\left|\int_{[s, T] \times H}\right| x\right|^{4}+|F(t, x)|^{2}+|x|^{4} \cdot|F(t, x)|^{2} \eta(\mathrm{d} t, \mathrm{~d} x)<\infty\right\} .
$$

The differences in the moment conditions are caused by our optimized upper bound achieved in Theorem 1; see also Remark 2.4.

It seems reasonable to expect, that existence results for solutions to Fokker-Planck equations, similar to those obtained in BDPR10], can also be established in the case of noise with jumps. See also Remark 6.2 below.

Remark 2.6. Similarly to the case of a measurable nonlinear drift part $F$, all main results of BDPR09 for (SPDE) with m-dissipative nonlinear drift term $F$ can be generalized to the case of (SPDE) driven by noise with jumps. In particular, mdissipativity of the Kolmogorov operator $L$ and uniqueness of solutions to (FPE) can be confirmed to hold within our framework. While the m-dissipativity condition on $F$ is more restrictive than (H.4), Hypothesis (H.5) can be dropped in this alternative case. Similarly to Theorem 2, we obtain relaxed integrability conditions on $\eta$ compared to BDPR09]. We refer to Wie11, Sect. 2.2.3] for details. 


\section{THE LINEAR CASE}

The objective of this section is the generalization of results from BDPR09 concerning the generator of $\left(S_{t}\right)$, to include the case of explicit time-dependence of the function space, on which $\left(S_{t}\right)$ acts. To this end, we adapt and extend methods and results from the literature on generalized Mehler semigroups (particularly, LR02 and (LR04]).

Throughout this section, we assume that Hypotheses (H.1) (H.3) hold.

3.1. The generalized Mehler semigroup $\left(S_{t}\right)$. We start with an integration by parts formula. Before we formulate the result, let us recall the following (see e.g. DP04, p.11f] for details): The range of $Q_{t}^{1 / 2}$ is a strict subset of $H: Q_{t}^{1 / 2}(H) \subsetneq H$. Thus, the white noise function $W$, given as

$$
\begin{aligned}
W: Q_{t}^{1 / 2}(H) & \rightarrow L^{2}\left(H, N_{Q_{t}}\right) \\
f \quad & \mapsto\left\langle\cdot, Q_{t}^{-1 / 2} f\right\rangle=: W_{f},
\end{aligned}
$$

is not defined on all of $H$. However, it can be extended uniquely to a mapping $H \rightarrow$ $L^{2}\left(H, N_{Q_{t}}\right)$. Even for this extension, we use the notation $W_{f}(\cdot)=\left\langle\cdot, Q_{t}^{-1 / 2} f\right\rangle$ for any $f \in H$. Note, that the extension of the white noise function for arbitrary arguments in $H$ requires, that $\operatorname{ker} Q_{t}=\{0\}$ (cf. (H.2)).

Lemma 3.1. For $\varphi \in \mathcal{C}_{u, 1}(H), h \in H, t \in(0, T]$, we have for all $x \in H$, that

$$
\begin{gathered}
\left\langle D S_{t} \varphi(\cdot)(x), h\right\rangle \\
\left.=\int_{H} \int_{H}(\int_{H} \varphi\left(e^{t A} x+y_{1}+y_{2}+y_{3}\right) \cdot \underbrace{Q_{t}^{-1 / 2} e^{t A}}_{=\Lambda_{t}} h, Q_{t}^{-1 / 2} y_{1}\rangle N_{Q_{t}}\left(\mathrm{~d} y_{1}\right)\right) \\
e_{G}\left(M_{t}\right)\left(\mathrm{d} y_{2}\right) \delta_{b_{t}}\left(\mathrm{~d} y_{3}\right) .
\end{gathered}
$$

The following proof adapts a result from the Wiener noise case to our framework; see e.g. [DP04, Cor. 1.6 and Prop. 1.7] or DPZ02, Thm. 6.2.2]. The main reason, why this works, is that the additional jump part in the noise does not affect the smoothing property of the diffusion part of the noise, which in turn is crucial for the argument.

Proof. Using the definition of $\left(S_{t}\right)$ and the decomposition $\mu_{t}=N_{Q_{t}} * e_{G}\left(M_{t}\right) * \delta_{b_{t}}$, we observe that

$$
\begin{gathered}
\left\langle D S_{t} \varphi(\cdot)(x), h\right\rangle \\
=\lim _{\varepsilon \rightarrow 0} \frac{1}{\varepsilon} \int_{H} \int_{H}\left(\int_{H} \varphi\left(e^{t A} x+y_{1}+y_{2}+y_{3}+\varepsilon \cdot e^{t A} h\right)-\varphi\left(e^{t A} x+y_{1}+y_{2}+y_{3}\right)\right. \\
\left.N_{Q_{t}}\left(\mathrm{~d} y_{1}\right)\right) e_{G}\left(M_{t}\right)\left(\mathrm{d} y_{2}\right) \delta_{b_{t}}\left(\mathrm{~d} y_{3}\right) \\
=\lim _{\varepsilon \rightarrow 0} \frac{1}{\varepsilon} \int_{H} \int_{H}\left(\int_{H} \varphi\left(e^{t A} x+y_{1}+y_{2}+y_{3}\right) \cdot\left(e^{\left\langle\Lambda_{t}(\varepsilon h), Q_{t}^{-1 / 2} y_{1}\right\rangle-\frac{1}{2}\left|\Lambda_{t}(\varepsilon h)\right|^{2}}-1\right)\right. \\
\left.N_{Q_{t}}\left(\mathrm{~d} y_{1}\right)\right) e_{G}\left(M_{t}\right)\left(\mathrm{d} y_{2}\right) \delta_{b_{t}}\left(\mathrm{~d} y_{3}\right),
\end{gathered}
$$

where we used the Cameron-Martin formula in the last step. Let us identify an $N_{Q_{t}}$-integrable upper bound (independent of $\varepsilon$ ) for the following term: By the 
intermediate value theorem, there is for any $\varepsilon \in(0,1]$ an $\varepsilon_{0} \in(0, \varepsilon)$, such that

$$
\begin{gathered}
\left|\frac{1}{\varepsilon} \cdot\left(\exp \left[\left\langle\varepsilon \cdot \Lambda_{t} h, Q_{t}^{-1 / 2} y_{1}\right\rangle-\frac{\varepsilon^{2}}{2} \cdot\left|\Lambda_{t} h\right|^{2}\right]-1\right)\right| \\
=\left.\left|\left\langle\Lambda_{t} h, Q_{t}^{-1 / 2} y_{1}\right\rangle-\varepsilon_{0}\right| \Lambda_{t} h\right|^{2} \mid \cdot \exp \left[\left\langle\varepsilon_{0} \cdot \Lambda_{t} h, Q_{t}^{-1 / 2} y_{1}\right\rangle-\frac{\varepsilon_{0}^{2}}{2} \cdot\left|\Lambda_{t} h\right|^{2}\right] \\
\leq(\underbrace{\left|\left\langle\Lambda_{t} h, Q_{t}^{-1 / 2} y_{1}\right\rangle\right|}_{\leq \exp \left[\left|\left\langle\Lambda_{t} h, Q_{t}^{-1 / 2} y_{1}\right\rangle\right|\right]}+\left|\Lambda_{t} h\right|^{2}) \cdot \exp \left[\left|\left\langle\Lambda_{t} h, Q_{t}^{-1 / 2} y_{1}\right\rangle\right|\right] \\
\leq\left(1+\left|\Lambda_{t} h\right|^{2}\right) \cdot \exp \left[2 \cdot\left|\left\langle\Lambda_{t} h, Q_{t}^{-1 / 2} y_{1}\right\rangle\right|\right] .
\end{gathered}
$$

Due to

$$
\begin{gathered}
\int_{H} \exp \left[2 \cdot\left|\left\langle\Lambda_{t} h, Q_{t}^{-1 / 2} y_{1}\right\rangle\right|\right] N_{Q_{t}}\left(\mathrm{~d} y_{1}\right) \\
\leq \int_{H} \exp \left[-2\left\langle\Lambda_{t} h, Q_{t}^{-1 / 2} y_{1}\right\rangle\right]+\exp \left[2\left\langle\Lambda_{t} h, Q_{t}^{-1 / 2} y_{1}\right\rangle\right] N_{Q_{t}}\left(\mathrm{~d} y_{1}\right)=2 e^{2\left|\Lambda_{t} h\right|^{2}}
\end{gathered}
$$

since $\left\langle\Lambda_{t} h, Q_{t}^{-1 / 2} y_{1}\right\rangle=W_{\Lambda_{t} h}\left(y_{1}\right)$ fulfills $N_{Q_{t}} \circ W_{\Lambda_{t} h}^{2} \sim N\left(0,\left|\Lambda_{t} h\right|^{2}\right)$ (cf. e.g. DP06, Prop. 1.15]), we may use Lebesgue's dominated convergence theorem to obtain, that

$$
\begin{gathered}
\left\langle D S_{t} \varphi(\cdot)(x), h\right\rangle \\
=\int_{H} \int_{H} \int_{H} \varphi\left(e^{t A} x+y_{1}+y_{2}+y_{3}\right) \cdot \lim _{\varepsilon \rightarrow 0} \frac{1}{\varepsilon}\left(e^{\varepsilon\left\langle\Lambda_{t} h, Q_{t}^{-1 / 2} y_{1}\right\rangle-\frac{\varepsilon^{2}}{2}\left|\Lambda_{t} h\right|^{2}}-1\right) \\
N_{Q_{t}}\left(\mathrm{~d} y_{1}\right) e_{G}\left(M_{t}\right)\left(\mathrm{d} y_{2}\right) \delta_{b_{t}}\left(\mathrm{~d} y_{3}\right),
\end{gathered}
$$

which proves the claim.

The following result and its proof are similar to the Wiener noise case (see DPZ02, Prop. 11.2.5] and [Cer95]). Only the integration by parts formula used in the proof is formulated differently (see Lemma 3.1 above), but the estimates remain the same.

Lemma 3.2. We have the following Feller properties for $\left(S_{t}\right)$ :

(i) For $\varphi \in \mathcal{C}_{u}(H)$ and all $(t, x) \in[0, T] \times H,\left|S_{t} \varphi(x)\right| \leq\|\varphi\|_{0}$.

(ii) For all $\varphi \in \mathcal{C}_{u, k}(H), k \in \mathbb{N}$ and $t \in[0, T]$, we have

$$
\left\|S_{t} \varphi\right\|_{u, k} \leq C e^{\omega t} \cdot\|\varphi\|_{u, k} \cdot \int_{H} 1+|y|^{k} \mu_{t}(\mathrm{~d} y),
$$

where $C \in(0, \infty)$ is independent of $t, x$ and $\varphi$.

(iii) For $\varphi \in \mathcal{C}_{u}(H)$ and all $(t, x) \in(0, T] \times H$,

$$
\left|D S_{t} \varphi(x)\right| \leq\left\|\Lambda_{t}\right\|_{L(H)} \cdot\|\varphi\|_{0} \text {. }
$$

(iv) For all $(t, x) \in(0, T] \times H$ and $\varphi \in \mathcal{C}_{u, 1}(H)$, we have

$$
\begin{aligned}
& \frac{\left|D S_{t} \varphi(x)\right|}{1+|x|} \leq \tilde{C} e^{t \omega} \cdot\left\|\Lambda_{t}\right\|_{L(H)} \cdot\left(\int_{H} 1+|y|^{2} \mu_{t}(\mathrm{~d} y)\right)^{1 / 2} \cdot\|\varphi\|_{u, 1}, \\
& \text { where } \tilde{C} \in(0, \infty) \text { is independent of } t, x \text { and } \varphi .
\end{aligned}
$$

Note, that our assumptions imply the existence of finite first and second moments for $\mu_{t}$. Thus, in our framework the upper bounds in (ii), if $k \in\{1,2\}$, and in (iv) are finite. 
3.2. The space-time homogenization of the generalized Mehler semigroup and its generator. Recall, that $V_{0} \psi(t, \cdot):=D_{t} \psi(t, \cdot)+U \psi(t, \cdot)$ for any $\psi \in$ $\mathcal{W}_{T, A}$. The following remark adapts LR02, Thm. 1.1(i)]) to the time-dependent case. The proof is similar to that in LR02, and thus not included. (The observation, that $U$ maps the test function space into itself, has not been made in LR02, but it follows immediately from the proof presented there.)

Remark 3.3. For $\psi \in \mathcal{W}_{T, A}$, we have $U \psi \in \mathcal{W}_{T, A}$ and $V_{0} \psi \in \mathcal{C}_{T}\left([0, T] ; \mathcal{C}_{u}(H)\right)$.

To extend the operator $V_{0}$ to a larger domain, we consider for each $\alpha \in \mathbb{R}$

$$
R_{\alpha}^{V} f(t, \cdot)(x):=\int_{t}^{T} e^{-\alpha(s-t)} \cdot S_{s-t} f(s, \cdot)(x) \mathrm{d} s, \quad f \in \mathcal{C}\left([0, T] ; \mathcal{C}_{u, 1}(H)\right) .
$$

By Lemma $3.2, R_{\alpha}^{V} f \in \mathcal{C}\left([0, T] ; \mathcal{C}_{u, 1}(H)\right)$ for any $f \in \mathcal{C}\left([0, T] ; \mathcal{C}_{u, 1}(H)\right)$.

Remark 3.4. $R_{\alpha}^{V}$ fulfills the resolvent identity

$$
R_{\alpha}^{V}-R_{\kappa}^{V}=(\kappa-\alpha) \cdot R_{\kappa}^{V} R_{\alpha}^{V} \quad \text { for all } \kappa, \alpha \in \mathbb{R} .
$$

As a consequence of Remark 3.4 it follows, that the range $R_{\alpha}^{V}\left(\mathcal{C}\left([0, T] ; \mathcal{C}_{u, 1}(H)\right)\right)$ does not depend on the choice of $\alpha$. We also observe, that for any $\varphi \in \mathcal{C}\left([0, T] ; \mathcal{C}_{u, 1}(H)\right)$

$$
\begin{aligned}
& \alpha \cdot R_{\alpha}^{V} \varphi(t, x)=\alpha \cdot \int_{0}^{T-t} e^{-\alpha s} \cdot S_{s} \varphi(s+t, \cdot)(x) \mathrm{d} s \\
= & \int_{0}^{\alpha(T-t)} e^{-s} \cdot S_{s / \alpha} \varphi\left(\frac{s}{\alpha}+t, \cdot\right)(x) \mathrm{d} s \stackrel{\alpha \rightarrow \infty}{\longrightarrow} \varphi(t, x) .
\end{aligned}
$$

Thus, $R_{\alpha}^{V}$ is injective and continuous for each $\alpha$, with $D\left(R_{\alpha}^{V}\right):=\mathcal{C}\left([0, T] ; \mathcal{C}_{u, 1}(H)\right)$. Consequently, for each $\alpha$ the inverse operator $\left(R_{\alpha}^{V}\right)^{-1}$ exists and is a closed linear operator on $R_{\alpha}^{V}\left(D\left(R_{\alpha}^{V}\right)\right)$. Which implies, that

$$
V:=\alpha I-\left(R_{\alpha}^{V}\right)^{-1}
$$

is a closed linear operator defined on

$$
D(V):=R_{\alpha}^{V}\left(\mathcal{C}\left([0, T] ; \mathcal{C}_{u, 1}(H)\right)\right) \quad\left(\subset \mathcal{C}\left([0, T] ; \mathcal{C}_{u, 1}(H)\right)\right) .
$$

(Again, this definition is independent of $\alpha$.)

It is easily seen, that the family $\left(S_{\tau}^{T}\right)_{\tau \geq 0}$ of operators given by the space-time homogenization of $\left(S_{t}\right)$ in the space $\mathcal{C}_{T}\left([0, T] ; \mathcal{C}_{u, 1}(H)\right)$, cf. Section 2 forms a semigroup. Observe furthermore, that for any $\varphi \in \mathcal{C}_{T}\left([0, T] ; \mathcal{C}_{u, 1}(H)\right)$ we have

$$
\int_{0}^{\infty} e^{-\alpha r} \cdot\left(S_{r}^{T} \varphi\right)(t, x) \mathrm{d} r=\int_{0}^{T-t} e^{-\alpha r} \cdot S_{r} \varphi(t+r, \cdot)(x) \mathrm{d} r=R_{\alpha}^{V} \varphi(t, \cdot)(x) .
$$

The next remark generalizes LR02, Thm. 1.3(i)] to the time-dependent case.

Remark 3.5. For any $\tau \in[0, T]$, we have $S_{\tau}^{T}\left(\mathcal{W}_{T, A}\right) \subset \mathcal{W}_{T, A}$ and $S_{\tau}\left(\mathcal{W}_{T, A}\right) \subset$ $\mathcal{W}_{T, A}$.

The proof is a more detailed and time-dependent version of that in LR02]. In the proof we denote by $B_{\tau, m}$ the diagonal $(m \times m)$-matrix

$$
B_{\tau, m}=\left(\begin{array}{ccc}
e^{\tau \alpha_{1}} & & 0 \\
& \ddots & \\
0 & & e^{\tau \alpha_{m}}
\end{array}\right), \quad \tau \in[0, T], m \in \mathbb{N},
$$


where $\left\{\alpha_{i}\right\}_{i \in \mathbb{N}}$ are the eigenvalues of $A$ corresponding to $\left\{\xi_{i}\right\}_{i \in \mathbb{N}}$ (cf. Rem. 2.1). Furthermore, we define $\phi_{\tau}:[0, T] \rightarrow \mathbb{R}$ by

$$
\phi_{\tau}(s):= \begin{cases}\phi(s+\tau) & \text { if } s+\tau \leq T \\ 0 & \text { if } s+\tau>T .\end{cases}
$$

Observe, that $\phi_{\tau} \in \mathcal{C}^{2}([0, T])$, and that $\phi_{\tau}(T)=\phi_{\tau}^{\prime}(T)=\phi_{\tau}^{\prime \prime}(T)=0$.

Proof of Remark 3.5. Let $\psi \in \mathcal{W}_{T, A}$ (of the form (1.3)) and $(s, \tau) \in[0, T] \times[0, T]$. Then,

$$
\begin{aligned}
& \left(S_{\tau}^{T} \psi\right)(s, x)= \begin{cases}0 & \text { if } s+\tau>T \\
\int_{H} \psi\left(s+\tau, e^{\tau A} x+y\right) \mu_{\tau}(\mathrm{d} y) & \text { if } s+\tau \leq T\end{cases} \\
& =\phi_{\tau}(s) \int_{H} \int_{\mathbb{R}^{m}} \exp \left[i\left\langle\Pi_{m}(r), e^{\tau A} x+y\right\rangle\right] \nu_{m}(\mathrm{~d} r) \mu_{\tau}(\mathrm{d} y) .
\end{aligned}
$$

Since the absolute value of the integrand is bounded by $1, \nu_{m} \in \mathcal{M}_{b}^{\mathbb{C}}\left(\mathbb{R}^{m}\right)$ and $\mu_{\tau} \in \mathcal{M}_{1}(H)$, we can apply Fubini's theorem to obtain that

$$
\begin{aligned}
&\left(S_{\tau}^{T} \psi\right)(s, x) \\
&= \phi_{\tau}(s) \int_{\mathbb{R}^{m}} \exp \left[i\left\langle\Pi_{m}(r), e^{\tau A} x\right\rangle\right] \cdot\left(\int_{H} \exp \left[i\left\langle\Pi_{m}(r), y\right\rangle\right] \mu_{\tau}(\mathrm{d} y)\right) g_{m}(r) \mathrm{d} r \\
&= \phi_{\tau}(s) \int_{\mathbb{R}^{m}} \underbrace{\exp \left[i \sum_{j=1}^{m} r_{j} \cdot\left\langle e^{\tau \alpha_{j}} \xi_{j}, x\right\rangle\right]}_{=e^{i\left(B_{\tau} r,\left(\left\langle\xi_{1}, x\right\rangle, \ldots,\left\langle\xi_{m}, x\right\rangle\right) \mathbb{R}^{m}\right.}} \cdot \underbrace{\exp \left[-\int_{0}^{\tau} \lambda\left(\sum_{j=1}^{m} r_{j} \cdot e^{u \alpha_{j}} \xi_{j}\right) \mathrm{d} u\right]}_{=\hat{\mu}\left(\Pi_{m}(r)\right)} g_{m}(r) \mathrm{d} r \\
&=\phi_{\tau}(s) \int_{\mathbb{R}^{m}}^{\exp \left[i\left(r, P_{m} x\right)_{\mathbb{R}^{m}}\right]} \cdot \underbrace{\hat{\mu}_{\tau}\left(\Pi_{m}\left(B_{\tau, m}^{-1} r\right)\right) g_{m}\left(B_{\tau, m}^{-1} r\right) \cdot \frac{1}{\operatorname{det} B_{\tau, m}}}_{=: \tilde{g}_{m}(r)} \mathrm{d} r .
\end{aligned}
$$

Due to the regularity properties of $\left.\lambda\right|_{F_{m}}$, we have that $\tilde{g}_{m}(r) \in \mathcal{S}\left(\mathbb{R}^{m} ; \mathbb{C}\right)$. The fact, that $S_{\tau}^{T} \psi$ is real-valued, follows by construction.

A similar argument shows that $S_{\tau}\left(\mathcal{W}_{T, A}\right) \subset \mathcal{W}_{T, A}$ as well.

Remark 3.6. The semigroup $\left(S_{\tau}^{T}\right)_{\tau \geq 0}$ is a $\pi$-semigroup on $\mathcal{C}_{T}\left([0, T] ; \mathcal{C}_{u, 1}(H)\right)$.

(In particular, continuity in time $\tau$ follows from an argument similar to the proof of [Man06, Prop 4.6(iii)].)

To show that $V$, as the extension of $V_{0}$, generates the semigroup $\left(S_{\tau}^{T}\right)_{\tau \geq 0}$ in the sense of $\pi$-semigroups (arguing as in Pri99]), we use the following criteria:

$$
\Leftrightarrow\left\{\begin{array}{c}
u \in D(V) \text { and } V u=f \\
\lim _{h \rightarrow 0} \frac{\left(S_{h}^{T} u\right)(t, x)-u(t, x)}{h}=f(t, x) \quad \text { for all }(t, x) \in[0, T] \times H \\
\sup _{\substack{h \in(0, T],(t, x) \in[0, T] \times H}} \frac{(1+|x|)^{-1}}{h} \cdot\left|\left(S_{h}^{T} u\right)(t, x)-u(t, x)\right|<\infty .
\end{array}\right.
$$

For the first condition, we generalize [LR02, Thm. 1.1(ii)] to the case of explicit time-dependence. 
Lemma 3.7. For any $\psi \in \mathcal{W}_{T, A}, x \in H, t \in[0, T)$ and $h \in[0, T)$ we have that

$$
\left(S_{h}^{T} \psi\right)(t, x)-\psi(t, x)=\int_{0}^{h}\left(S_{s}^{T} V_{0} \psi\right)(t, x) \mathrm{d} s .
$$

Proof. Assume without loss of generality, that $\psi \in \mathcal{W}_{T, A}$ is of the form (1.3). By Remark 3.3 , we know that $V_{0} \psi \in \mathcal{C}_{T}\left([0, T] ; \mathcal{C}_{u}(H)\right)$ for all $\psi \in \mathcal{W}_{T, A}$. Thus, $S_{s}^{T} V_{0} \psi$ is well-defined, and by the definitions of $S_{s}^{T}$ and $V_{0}$ we see that

$$
\left(S_{s}^{T} V_{0} \psi\right)(t, x)= \begin{cases}S_{s} V_{0} \psi(t+s, \cdot)(x) & \text { if } s \leq T-t \\ 0 & \text { if } s+t>T\end{cases}
$$

(from here on, assume first that the first case holds)

$$
\begin{gathered}
=\int_{H} V_{0} \psi\left(t+s, e^{s A} x+y\right) \mu_{s}(\mathrm{~d} y) \\
=\int_{H} U \psi\left(t+s, e^{s A} x+y\right) \mu_{s}(\mathrm{~d} y)+\int_{H}\left(D_{t} \psi\right)\left(t+s, e^{s A} x+y\right) \mu_{s}(\mathrm{~d} y) .
\end{gathered}
$$

We start by considering the summands separately.

Similarly to [LR02, pp. 303-305] we obtain, that

$$
\begin{gathered}
\int_{0}^{h} \int_{H} U \psi\left(t+s, e^{s A} x+y\right) \mu_{s}(\mathrm{~d} y) \mathrm{d} s \\
=\int_{0}^{h} \int_{H}\left(i\left\langle e^{s A} A \xi, x\right\rangle-\lambda\left(e^{s A} \xi\right)\right) \\
\cdot \exp \left[i\left\langle e^{s A} \xi, x\right\rangle-\int_{0}^{s} \lambda\left(e^{\theta A} \xi\right) \mathrm{d} \theta\right] \nu_{t+s}(\mathrm{~d} \xi) \mathrm{d} s \\
=\int_{0}^{h} \int_{\mathbb{R}^{m}} \frac{\mathrm{d}}{\mathrm{d} s}\left(\exp \left[i\left\langle e^{s A}\left(\sum_{j=1}^{m} r_{j} \xi_{j}\right), x\right\rangle-\int_{0}^{s} \lambda\left(e^{\theta A}\left(\sum_{j=1}^{m} r_{j} \xi_{j}\right)\right) \mathrm{d} \theta\right]\right) \\
\cdot \phi(s+t) \cdot g_{m}(r) \mathrm{d} r \mathrm{~d} s .
\end{gathered}
$$

After applying Fubini's Theorem we see, that for each fixed $r \in \mathbb{R}^{m}$

$$
\begin{gathered}
\int_{0}^{h} \frac{\mathrm{d}}{\mathrm{d} s}\left(\exp \left[i\left\langle e^{s A}\left(\sum_{j=1}^{m} r_{j} \xi_{j}\right), x\right\rangle-\int_{0}^{s} \lambda\left(e^{\theta A}\left(\sum_{j=1}^{m} r_{j} \xi_{j}\right)\right) \mathrm{d} \theta\right]\right) \cdot \phi(s+t) \cdot g_{m}(r) \mathrm{d} s \\
=\left[\exp \left[i\left\langle e^{s A}\left(\sum_{j=1}^{m} r_{j} \xi_{j}\right), x\right\rangle-\int_{0}^{s} \lambda\left(e^{\theta A}\left(\sum_{j=1}^{m} r_{j} \xi_{j}\right)\right) \mathrm{d} \theta\right] \cdot \phi(s+t) \cdot g_{m}(r)\right]_{s=0}^{s=h} \\
-\int_{0}^{h} \exp \left[i\left\langle e^{s A}\left(\sum_{j=1}^{m} r_{j} \xi_{j}\right), x\right\rangle-\int_{0}^{s} \lambda\left(e^{\theta A}\left(\sum_{j=1}^{m} r_{j} \xi_{j}\right)\right) \mathrm{d} \theta\right] \\
\cdot\left(\frac{\mathrm{d}}{\mathrm{d} s} \phi(t+s)\right) \cdot g_{m}(r) \mathrm{d} s
\end{gathered}
$$


For the time derivative summand in 3.2 , we observe that

$$
\begin{gathered}
\int_{H}\left(D_{t} \psi\right)\left(t+s, e^{s A} x+y\right) \mu_{s}(\mathrm{~d} y) \\
=\int_{H} D_{t}\left(\phi(t+s) \cdot f_{m}\left(\left\langle\xi_{1}, e^{s A} x+y\right\rangle, \ldots,\left\langle\xi_{m}, e^{s A} x+y\right\rangle\right)\right) \mu_{s}(\mathrm{~d} y) \\
=\left(\frac{\mathrm{d}}{\mathrm{d} t} \phi(t+s)\right) \cdot \int_{H} \int_{\mathbb{R}^{m}} \exp \left[i\left(\sum_{j=1}^{m} r_{j}\left\langle\xi_{j}, e^{s A} x+y\right\rangle\right)\right] g_{m}(r) \mathrm{d} r \mu_{s}(\mathrm{~d} y) \\
=\left(\frac{\mathrm{d}}{\mathrm{d} t} \phi(t+s)\right) \cdot \int_{\mathbb{R}^{m}} \int_{H} \exp \left[i\left\langle\left(\sum_{j=1}^{m} r_{j} \xi_{j}\right), y\right\rangle\right] \mu_{s}(\mathrm{~d} y) \cdot \exp \left[i\left(\sum_{j=1}^{m} r_{j}\left\langle\xi_{j}, e^{s A} x\right\rangle\right)\right] \\
=\left(\frac{\mathrm{d}}{\mathrm{d} t} \phi(t+s)\right) \cdot \int_{\mathbb{R}^{m}} \exp \left[-\int_{0}^{s} \lambda\left(e^{\theta A}\left(\sum_{j=1}^{m} r_{j} \xi_{j}\right)\right) \mathrm{d} \theta+i\left(\sum_{j=1}^{m} r_{j}\left\langle\xi_{j}, e^{s A} x\right\rangle\right)\right]
\end{gathered}
$$

Finally, using (3.3), (3.4) and the equation above we obtain:

$$
\begin{gathered}
\int_{0}^{h}\left(S_{s}^{T} V_{0} \psi\right)(t, x) \mathrm{d} s \\
=\int_{\mathbb{R}^{m}}\left[\exp \left[i\left\langle e^{s A}\left(\sum_{j=1}^{m} r_{j} \xi_{j}\right), x\right\rangle-\int_{0}^{s} \lambda\left(e^{\theta A}\left(\sum_{j=1}^{m} r_{j} \xi_{j}\right)\right) \mathrm{d} \theta\right]\right. \\
\left.\cdot \phi(s+t) \cdot g_{m}(r)\right]_{s=0}^{s=h} \mathrm{~d} r \\
=\phi(h+t) \cdot \int_{\mathbb{R}^{m}} \exp \left[i\left\langle e^{h A}\left(\sum_{j=1}^{m} r_{j} \xi_{j}\right), x\right\rangle-\int_{0}^{h} \lambda\left(e^{\theta A}\left(\sum_{j=1}^{m} r_{j} \xi_{j}\right)\right) \mathrm{d} \theta\right] g_{m}(r) \mathrm{d} r \\
-\phi(t) \cdot \int_{\mathbb{R}^{m}} \exp \left[i\left\langle\left(\sum_{j=1}^{m} r_{j} \xi_{j}\right), x\right\rangle\right] g_{m}(r) \mathrm{d} r .
\end{gathered}
$$

Considering the two summands separately we see, that

$$
\phi(t) \cdot \int_{\mathbb{R}^{m}} \exp \left[i\left\langle\left(\sum_{j=1}^{m} r_{j} \xi_{j}\right), x\right\rangle\right] g_{m}(r) \mathrm{d} r=\psi(t, x)
$$

and, using Remark 1.1 and the different re-formulations of $\hat{\mu}_{h}(\xi)$,

$$
\begin{gathered}
\int_{H} \exp \left[i\left\langle e^{h A} \xi, x\right\rangle-\int_{0}^{h} \lambda\left(e^{\theta A} \xi\right) \mathrm{d} \theta\right] \nu_{h+t}(\mathrm{~d} \xi) \\
=\int_{H} \int_{H} \exp \left[i\left\langle\xi, e^{h A} x+y\right\rangle\right] \nu_{h+t}(\xi) \mu_{h}(\mathrm{~d} y)=S_{h} \psi(t+h, \cdot)(x) .
\end{gathered}
$$

Recalling that $\left(S_{h}^{T} \psi\right)(t, x)-\psi(t, x)=S_{h} \psi(t+h, \cdot)(x)-\psi(t, x)$ for $h \in[0, T)$, we conclude the proof for the case $s \leq T-t$.

Now, let $s+t>T$. Then, by definition of the family $\left(S_{\tau}^{T}\right)_{\tau \geq 0}$,

$$
\left(S_{s}^{T} \psi\right)(t, x)-\psi(t, x)=-\psi(t, x),
$$


whereas on the other hand, using the definitions and the result proved above for the case $s+t \leq T$

$$
\begin{gathered}
\int_{0}^{s}\left(S_{r}^{T} V_{0} \psi\right)(t, x) \mathrm{d} r=\int_{0}^{T-t}\left(S_{r}^{T} V_{0} \psi\right)(t, x) \mathrm{d} r+0 \\
=\left(S_{T-t}^{T} \psi\right)(t, x)-\psi(t, x)=S_{T-t} \psi(T, x)-\psi(t, x)=-\psi(t, x),
\end{gathered}
$$

which proves the assertion.

For the second criterium in (3.1) we show the following, stronger result (which actually implies that $\left(S_{h}^{T}\right)_{h \geq 0}$, restricted to $\mathcal{W}_{T, A}$, is a $C_{0}$-semigroup):

Lemma 3.8. For all $\psi \in \mathcal{W}_{T, A}$,

$$
\sup _{\substack{h \in(0, T],(t, x) \in[0, T] \times H}} \frac{\left|\left(S_{h}^{T} \psi\right)(t, x)-\psi(t, x)\right|}{h}<\infty .
$$

Proof. If $t=T$, then by definition of $\mathcal{W}_{T, A}$ we have that $\left(S_{h}^{T} \psi\right)(t, x)=\psi(t, x)=0$, and the claim is fulfilled. From here on, let $t<T$.

As before, assume first that $t+h \leq T$. Choose $\psi \in \mathcal{W}_{T, A}$ and assume without loss of generality, that $\psi(t, x)=\phi(t) \cdot f_{m}\left(P_{m} x\right)$. If $\psi=0$, the assertion is trivially fulfilled; assume that $\|\psi\|_{0, T}>0$. Use Lemma 3.7 to see that

$$
\left(S_{h}^{T} \psi\right)(t, x)-\psi(t, x)=\int_{0}^{h}\left(S_{s}^{T} V_{0} \psi\right)(t, x) \mathrm{d} s \leq h \cdot \sup _{s \in[0, h]}\left|\left(S_{s}^{T} V_{0} \psi\right)(t, x)\right| .
$$

By definition of $\left(S_{t}^{T}\right)_{t \geq 0}$ (and recalling that $t+h \leq T$, i.e. $s+t \leq T$ for $s \in[0, h]$ ),

$$
\left(S_{h}^{T} \psi\right)(t, x)-\psi(t, x) \leq h \cdot \sup _{s \in[0, h]}\left|S_{s} V_{0} \psi(t+s, \cdot)(x)\right| .
$$

Recall that, using Remark 3.3 there is a $\tilde{\psi} \in \mathcal{W}_{T, A}$, such that

$$
V_{0} \psi(t+s, \cdot)(x)=\left(D_{t}+U\right) \psi(t+s, \cdot)(x)=\phi^{\prime}(t+s) \cdot f_{m}\left(P_{m} x\right)+\tilde{\psi}(t+s, x) .
$$

Now we can use Lemma 3.2 (i) to obtain, that

$$
\sup _{x \in H}\left|\left(S_{h}^{T} \psi\right)(t, x)-\psi(t, x)\right| \leq h \cdot \underbrace{\sup _{(t, x) \in[0, T] \times H}\left|\phi^{\prime}(t) \cdot f_{m}\left(P_{m} x\right)+\tilde{\psi}(t, x)\right|}_{=: C},
$$

where $C \in(0, \infty)$ is independent of $h, s, t$ and $x$.

Consider the case $t+h>T$. There exists an $\varepsilon>0$, such that $h>\varepsilon$, and by definition of $\left(S_{h}^{T}\right)_{h \geq 0}$,

$$
\left(S_{h}^{T} \psi\right)(t, x)-\psi(t, x)=-\psi(t, x),
$$

which proves the claim, since $h>\varepsilon$ and $\mathcal{W}_{T, A} \subset \mathcal{C}\left([0, T] ; \mathcal{C}_{u}(H)\right)$.

3.3. A core for the generator. The following result and proof is adapted from BDPR09, Prop. A.2], which in turn generalizes [DPT01, Prop. 2.5]. Note, that we are working on a different space of test functions, which changes some of the arguments (in particular, the continuity argument; cf. Lemma 3.10 and (3.14)). As a consequence of these changes we gain, that the upper bounds (3.7) and (3.17) depend only linearly (and not quadratically) on $|x|$. 
Proposition 3.9. Let $u \in D(V), \varepsilon>0$, and $\eta$ a finite nonnegative Borel measure on $[0, T] \times H$. Then there exist a sequence $\left(\psi_{n}\right) \subset \mathcal{W}_{T, A}$, a constant $c \in(0, \infty)$ and an $n_{0} \in \mathbb{N}$, such that

$$
\left|\psi_{n}(t, x)\right|+\left|V_{0} \psi_{n}(t, x)\right| \leq(c T+1) \cdot\left(\|V u\|_{u, 1, T}+1\right) \cdot(1+|x|)
$$

for all $(t, x) \in[0, T] \times H$ and $n \geq n_{0}$, and

$$
\psi_{n} \stackrel{n \rightarrow \infty}{\longrightarrow} u \quad \text { and } \quad V \psi_{n}=V_{0} \psi_{n} \stackrel{n \rightarrow \infty}{\longrightarrow} V u
$$

converge in measure $\eta$ on $[0, T) \times H$.

Proof. Replacing $\eta$ by $\frac{1}{1+|x|} \cdot \eta$ we may assume, that $\int_{H} 1+|x| \eta(\mathrm{d} t, \mathrm{~d} x)<\infty$.

Let $f \in \mathcal{C}\left([0, T] ; \mathcal{C}_{u, 1}(H)\right)$ and $u=-R_{0}^{V} f=V^{-1} f$, i.e., for all $(t, x) \in[0, T] \times H$,

$$
u(t, x)=-\int_{t}^{T} S_{s-t} f(s, \cdot)(x) \mathrm{d} s .
$$

Note that, by definition of $V$, all $u \in D(V)$ are of this form.

By Lemma 1.2 we can identify a triple-index sequence $\left(\psi_{n_{1}, n_{2}, n_{3}}\right)_{n_{1}, n_{2}, n_{3} \in \mathbb{N}} \subset$ $\mathcal{W}_{T, A}$, such that for all $(t, x) \in[0, T) \times H$

$$
\begin{gathered}
\lim _{n_{1} \rightarrow \infty} \lim _{n_{2} \rightarrow \infty} \lim _{n_{3} \rightarrow \infty} \psi_{n_{1}, n_{2}, n_{3}}(t, x)=f(t, x) \text { and } \\
\left|\psi_{n_{1}, n_{2}, n_{3}}(t, x)\right| \leq\left(\|f\|_{u, 1, T}+1\right) \cdot(1+|x|) \quad \text { for all } n_{1}, n_{2}, n_{3} .
\end{gathered}
$$

To simplify notation, we denote the triple-index $n_{1}, n_{2}, n_{3}$ by $\bar{n}$ and the triple-limit $\lim _{n_{1} \rightarrow \infty} \lim _{n_{2} \rightarrow \infty} \lim _{n_{3} \rightarrow \infty}$ by $\lim _{\bar{n} \rightrightarrows \infty}$ for the rest of this proof; 3.8 now reads:

$$
\begin{gathered}
\lim _{\bar{n} \rightrightarrows \infty} \psi_{\bar{n}}(t, x)=f(t, x) \quad \text { and } \\
\left|\psi_{\bar{n}}(t, x)\right| \leq\left(\|f\|_{u, 1, T}+1\right) \cdot(1+|x|) \quad \forall \bar{n} .
\end{gathered}
$$

Now we set, for each $\bar{n}$ and all $(t, x) \in[0, T] \times H$,

$$
\begin{aligned}
& u_{\bar{n}}(t, x):=V^{-1} \psi_{\bar{n}}(t, x)=-\int_{t}^{T} S_{s-t} \psi_{\bar{n}}(s, \cdot)(x) \mathrm{d} s \\
& =-(T-t) \cdot \int_{0}^{1} S_{(T-t) r} \psi_{\bar{n}}((T-t) r+t, \cdot)(x) \mathrm{d} r .
\end{aligned}
$$

Again from Lemma 1.2 we conclude, that there exists a $c \in(0, \infty)$ independent of $t$ and $x$, such that for all $(t, x) \in[0, T) \times H$

$$
\begin{gathered}
\lim _{\bar{n} \rightrightarrows \infty} u_{\bar{n}}(t, x)=u(t, x) \text { and } \\
\frac{\left|u_{\bar{n}}(t, x)\right|}{1+|x|} \leq(T-t) \cdot \sup _{s \in[t, T]}\left(\frac{S_{s-t} \psi_{\bar{n}}(s, \cdot)(x)}{1+|x|}\right) \\
\leq T \cdot \sup _{s \in[t, T]}\left(\int_{H} \frac{\psi_{\bar{n}}\left(s, e^{(s-t) A} x+y\right)}{1+\left|e^{(s-t) A} x+y\right|} \cdot \frac{1+\left|e^{(s-t) A} x+y\right|}{1+|x|} \mu_{s-t}(\mathrm{~d} y)\right) \\
\leq c T \cdot\left(\|f\|_{u, 1, T}+1\right)=c T \cdot\left(\|V u\|_{u, 1, T}+1\right) \quad \forall \bar{n} .
\end{gathered}
$$

Furthermore, $V u_{\bar{n}}(t, x)=V V^{-1} \psi_{\bar{n}}(t, x)=\psi_{\bar{n}}$, hence by (3.8)

$$
\begin{gathered}
\lim _{\bar{n} \rightrightarrows \infty} V u_{\bar{n}}(t, x)=V u(t, x)=f(t, x) \quad \text { and } \\
\left|V u_{\bar{n}}(t, x)\right|=\left|\psi_{\bar{n}}(t, x)\right| \\
\leq\left(\|f\|_{u, 1, T}+1\right) \cdot(1+|x|)=\left(\|V u\|_{u, 1, T}+1\right) \cdot(1+|x|) \forall \bar{n} .
\end{gathered}
$$


Next, we construct sequences of elements of $\mathcal{W}_{T, A}$, which approximate the $u_{\bar{n}}$ (which in turn are elements of $\left.R_{\alpha}^{V}\left(\mathcal{W}_{T, A}\right) \subset \mathcal{C}\left([0, T] ; \mathcal{C}_{u}(H)\right)\right)$. We set

$$
\begin{aligned}
& \Sigma:=\left\{\text { partitions } \sigma_{N}=\left\{t_{0}, \ldots, t_{N}\right\} \text { of }[0,1] \mid 0=t_{0}<t_{1}<\cdots<t_{N}=1\right\} \\
&\left|\sigma_{N}\right|:=\max _{i=1, \ldots, N}\left|t_{i}-t_{i-1}\right| .
\end{aligned}
$$

For any given $\sigma_{N}=\left\{t_{0}, \ldots, t_{N}\right\} \in \Sigma$, triple-index $\bar{n}$ and $(t, x) \in[0, T] \times H$ we set

$$
u_{\bar{n}, \sigma_{N}}(t, x):=-(T-t) \cdot \sum_{k=1}^{N} S_{(T-t) t_{k}} \psi_{\bar{n}}\left((T-t) t_{k}+t, \cdot\right)(x) \cdot\left(t_{k}-t_{k-1}\right) .
$$

By Remark 3.5. $S_{t}\left(\mathcal{W}_{T, A}\right)$ is a subset of $\mathcal{W}_{T, A}$, and $S_{t} \psi$ depends on the same $A$ eigenspaces as $\psi$. Thus, the sum in the definition of $u_{\bar{n}, \sigma_{N}}$ is still in $\mathcal{W}_{T, A}$. Consider furthermore

$$
V_{0} u_{\bar{n}, \sigma_{N}}(t, x):=-(T-t) \cdot \sum_{k=1}^{N} S_{(T-t) t_{k}} V_{0} \psi_{\bar{n}}\left((T-t) t_{k}+t, \cdot\right)(x) \cdot\left(t_{k}-t_{k-1}\right) .
$$

(3.8), 3.9) and (3.11) together imply that for all $(t, x) \in[0, T) \times H$

$$
\lim _{\bar{n} \rightrightarrows \infty} \lim _{\left|\sigma_{N}\right| \rightarrow 0} u_{\bar{n}, \sigma_{N}}(t, x)=u(t, x) \text { and }
$$

$\left|u_{\bar{n}, \sigma_{N}}(t, x)\right| \leq c T \cdot\left(\|V u\|_{u, 1, T}+1\right) \cdot(1+|x|) \quad$ for all $\bar{n}$ and any $N$ large enough and similarly

$$
\lim _{\bar{n} \rightrightarrows \infty} \lim _{\left|\sigma_{N}\right| \rightarrow 0} V_{0} u_{\bar{n}, \sigma_{N}}(t, x)=V u(t, x) .
$$

By Lemma 3.10 below, the mapping $(t, s) \mapsto S_{s} \psi(t, \cdot)(x)$ is continuous in the topology of $\overline{\mathcal{C}_{u}(H)}$ for any $\psi \in \mathcal{W}_{T, A}$. Consequently,

$$
u_{\bar{n}, \sigma_{N}}(t, x) \stackrel{\left|\sigma_{N}\right| \rightarrow 0}{\longrightarrow} \underbrace{-(T-t) \cdot \int_{0}^{1} S_{(T-t) r} \psi_{\bar{n}}((T-t) r+t, \cdot)(x) \mathrm{d} r}_{=u_{\bar{n}}(t, x)}
$$

converges in the topology of $\mathcal{C}_{u}(H)$ for each $t \in[0, T]$. Thus, there is a $\delta>0$, such that if $\left|\sigma_{N}\right|<\delta$, then for all $\bar{n}, t, x$ and all $N$ big enough,

$$
\left|V u_{\bar{n}}(t, x)-\left(-(T-t) \cdot \sum_{k=1}^{N} S_{(T-t) t_{k}} V_{0} \psi_{\bar{n}}\left((T-t) t_{k}+t, \cdot\right)(x) \cdot\left(t_{k}-t_{k-1}\right)\right)\right| \leq 1
$$

which is equivalent to

$$
\underbrace{\mid V u_{\bar{n}, \sigma_{N}}(t, x)}_{=V_{0} u_{\bar{n}}, \sigma_{N}}|\leq| V u_{\bar{n}}(t, x) \mid+1 \text { for all } \bar{n}, t, x \text { and all } N \text { big enough. }
$$

Now, let $\sigma_{N} \in \Sigma$ be chosen as $\sigma_{N}=\left\{0,1 / 2^{N}, 2 / 2^{N}, \ldots, 1\right\}$. Clearly, $\left|\sigma_{N}\right| \stackrel{N \rightarrow \infty}{\longrightarrow}$ 0 . For $\bar{n}$ fixed, $u_{\bar{n}, \sigma_{N}}(t, x) \stackrel{N \rightarrow \infty}{\longrightarrow} u_{\bar{n}}(t, x)$. By (3.9) and (3.15), the pointwise convergences in (3.12) and 3.13 imply $L^{1}(\eta)$-convergence on $[0, T) \times H$ in both cases through the dominated convergence theorem of Lebesgue. Finally, we choose a sequence of elements $\psi_{n}$ from the net $u_{\bar{n}, \sigma_{N}}$, which preserves the convergences of $\psi_{n}$ and $V_{0} \psi_{n}$ to $u$ and $V u$, respectively, in $L^{1}(\eta)$ and thus in measure $\eta$. Without loss of generality, this sequence can be chosen such, that for an $n_{0}$ big enough

$$
\left|V_{0} \psi_{n}(t, x)\right| \leq\left(\|V u\|_{u, 1, T}+1\right) \cdot(1+|x|) \text { for all } t, x \text { and all } n \geq n_{0}
$$


(using (3.15) and (3.10).

Lemma 3.10. The mapping

$$
\begin{array}{cl}
{[0, T] \times[0, T]} & \rightarrow \mathcal{C}_{u}(H) \\
(t, s) & \mapsto S_{s} \psi(t, \cdot)
\end{array}
$$

is continuous in the topology of $\mathcal{C}_{u}(H)$ for all $\psi \in \mathcal{W}_{T, A}$.

Proof. Fix $(s, t) \in[0, T] \times[0, T]$, a test function $\psi \in \mathcal{W}_{T, A}$ and a sequence $\left(\left(s_{n}, t_{n}\right)\right)_{n \in \mathbb{N}}$ converging to $(s, t)$ as $n \rightarrow \infty$. Assume without loss of generality, that $\psi(t, x)$ is of the form $\phi(t) \cdot f_{m}\left(P_{m} x\right)$. We show that

$$
\lim _{n \rightarrow \infty} \sup _{x \in H}\left|S_{s_{n}} \psi\left(t_{n}, \cdot\right)(x)-S_{s} \psi(t, \cdot)(x)\right|=0 .
$$

Observe that

$$
\begin{gathered}
\left\|S_{s_{n}} \psi\left(t_{n}, \cdot\right)-S_{s} \psi(t, \cdot)\right\|_{0} \\
\leq\left\|S_{s_{n}} \psi\left(t_{n}, \cdot\right)-S_{s_{n}} \psi(t, \cdot)\right\|_{0}+\left\|S_{s_{n}} \psi(t, \cdot)-S_{s} \psi(t, \cdot)\right\|_{0} .
\end{gathered}
$$

We have

$$
\begin{gathered}
\left\|S_{s_{n}} \psi\left(t_{n}, \cdot\right)-S_{s_{n}} \psi(t, \cdot)\right\|_{0} \\
\leq\left|\phi\left(t_{n}\right)-\phi(t)\right| \cdot\left\|\int_{H} f_{m}\left(P_{m}\left(e^{s_{n} A} \cdot+y\right)\right) \mu_{s_{n}}(\mathrm{~d} y)\right\|_{0} \stackrel{n \rightarrow \infty}{\longrightarrow} 0,
\end{gathered}
$$

since $\phi \in \mathcal{C}^{2}([0, T])$ and $\sup _{z \in H} f_{m}\left(P_{m} z\right)<\infty$.

For the second summand on the right hand side of (3.16), by the semigroup property of $\left(S_{t}\right)$ it is sufficient to consider the case $s=0$ (i.e., $s_{n} \stackrel{n \rightarrow \infty}{\longrightarrow} 0$ ):

$$
\begin{gathered}
\left\|S_{s_{n}} \psi(t, \cdot)-S_{0} \psi(t, \cdot)\right\|_{0} \\
\leq\left\|S_{s_{n}} \psi(t, \cdot)-S_{s_{n}} \psi\left(t+s_{n}, \cdot\right)\right\|_{0}+\left\|S_{s_{n}}^{T} \psi(t, \cdot)-\psi(t, \cdot)\right\|_{0} .
\end{gathered}
$$

Here, the first summand on the right hand side converges to 0 as $n \rightarrow \infty$ by the same argument as above, and for the second summand we obtain convergence to 0 using Lemma 3.8 (resp., equation (3.6) in its proof).

Lemma 3.11. If $u \in D(V)$, then it is differentiable in space for all $t \in[0, T]$, and

$$
D u(t, x)=-\int_{t}^{T} D S_{s-t} V u(s, \cdot)(x) \mathrm{d} s .
$$

Proof. Let $u \in D(V)=R_{\alpha}^{V}\left(\mathcal{C}\left([0, T] ; \mathcal{C}_{u, 1}(H)\right)\right)$. Then there is an $f \in \mathcal{C}\left([0, T] ; \mathcal{C}_{u, 1}(H)\right)$ with $f=V u$, and we can write $u$ as

$$
u(t, x)=-\int_{t}^{T} S_{s-t} f(s, \cdot)(x) \mathrm{d} s .
$$

Recall that, by Lemma 3.2 , there is a $c \in(0, \infty)$ independent of $t$ and $x$, such that

$$
\left|D S_{\theta} f(t, x)\right| \leq c\left\|\Lambda_{\theta}\right\|_{L(H)} \cdot\left(\int_{H} 1+|y|^{2} \mu_{\theta}(\mathrm{d} y)\right)^{1 / 2} \cdot\|f(t, \cdot)\|_{u, 1} \cdot(1+|x|)
$$

for any $\theta>0, x \in H$ and $f \in \mathcal{C}\left([0, T] ; \mathcal{C}_{u, 1}(H)\right)$. Thus, integration and differentiation may be exchanged by (H.3) 
Corollary 3.12. Let $u \in D(V)$ and $\eta$ a finite nonnegative Borel measure on $[0, T] \times$ $H$.

Then, there exists a sequence $\left(\psi_{n}\right) \subset \mathcal{W}_{T, A}$, such that for a $c \in(0, \infty)$ and an $n_{0} \in \mathbb{N}$ large enough (similar to Proposition 3.9 above), we have

$$
\begin{gathered}
\left|\psi_{n}(t, x)\right|+\left|D \psi_{n}(t, x)\right|+\left|V_{0} \psi_{n}(t, x)\right| \\
\leq\left(c T+1+\int_{0}^{T}\left\|\Lambda_{s}\right\|_{L(H)} \mathrm{d} s\right) \cdot\left(\|V u\|_{u, 1, T}+1\right) \cdot(1+|x|)
\end{gathered}
$$

for all $(t, x) \in[0, T] \times H$ and $n \geq n_{0}$, and $\psi_{n} \rightarrow u,\left\langle D \psi_{n}, h\right\rangle \rightarrow\langle D u, h\rangle, V_{0} \psi_{n} \rightarrow V u$ converge in measure $\eta$ as $n \rightarrow \infty$ for any $h \in H$.

Observe that $\int_{0}^{T}\left\|\Lambda_{s}\right\|_{L(H)} \mathrm{d} s<\infty$ by Hypothesis (H.3).

Proof. Let $\left(\psi_{n}\right)$ be the approximating sequence constructed in Proposition 3.9 above. By Lemma 3.2, Lemma 3.11 and Proposition 3.9, we have

$$
D \psi_{n}(t, x) \leq \int_{0}^{T}\left\|\Lambda_{s}\right\|_{L(H)} \mathrm{d} s \cdot\left(\|V u\|_{u, 1, T}+1\right) \cdot(1+|x|)
$$

for all $(t, x) \in[0, T] \times H$ and any $n \in \mathbb{N}$ big enough. Thus, the claimed upper bound is valid in light of the proposition.

It remains to show the convergence of $\left\langle D \psi_{n}, h\right\rangle \rightarrow\langle D u, h\rangle$ as stated in the claim. We use the convergence result in the proposition, that $V_{0} \psi_{n} \rightarrow V u$ converges in measure $\eta$ on $[0, T) \times H$. Applying the integration by parts formula in Lemma 3.1 together with Lemma 3.2 and Lemma 3.11. we see that for each $h \in H,(t, x) \in$ $[0, T) \times H$,

$$
\begin{gathered}
\lim _{n \rightarrow \infty}\left\langle D \psi_{n}(t, x), h\right\rangle=\left\langle-\lim _{n \rightarrow \infty} \int_{t}^{T} D S_{s-t} V_{0} \psi_{n}(s, x) \mathrm{d} s, h\right\rangle \\
=\left\langle-\int_{t}^{T} D S_{s-t} V u(s, x) \mathrm{d} s, h\right\rangle=\langle D u(t, x), h\rangle .
\end{gathered}
$$

\section{REgular DRIFT}

Before we consider (SPDE) in the singular case, we need to collect some observations for the case, where the following regularity condition is fulfilled. These observations adapt parts of [BDPR09, Sect. 2] to our framework. Let us note that, actually, all main results of BDPR09, Sect. 2] can be generalized to our framework for noise with jumps; we refer to Wie11.

For this section we assume, that in addition to (H.1) (H.3) the following holds:

(H.6) Both $F:[0, T] \times H \rightarrow H$ and $D F(t, \cdot): H \rightarrow L(H)$ (the latter for any $t \in[0, T])$ are continuous.

Furthermore, there is a $K>0$, such that

$$
|F(t, x)-F(t, y)| \leq K \cdot|x-y| \text { for all } x, y \in H, t \in[0, T] .
$$

We use the following fact (e.g. from MPR10, Thm. 2.4], where actually even the multiplicative case is covered; see also [MR10, Thm. 12 and Rem. 13]):

Remark 4.1. Given Hypotheses (H.1) (H.3) and (H.6), (SPDE) has for any $s \geq 0$ a mild solution $(X(t, s, x))_{s \leq t \leq T}$ with cadlag sample paths, given by

$$
X(t, s, x)=e^{(t-s) A} x+\int_{s}^{t} e^{(t-r) A} F(r, X(r, s, x)) \mathrm{d} r+\int_{s}^{t} e^{(t-r) A} \mathrm{~d} Y(r)
$$


for all $0 \leq s \leq t \leq T$. The map $x \mapsto X(t, s, x)$ is Lipschitz continuous, and the solution has the Markov property.

Define the transition evolution operator related to (SPDE) by

$$
P_{s, t} \varphi(x):=\mathbb{E}[\varphi(X(t, s, x))], \quad 0 \leq s \leq t \leq T, \varphi \in \mathcal{C}_{u}(H) .
$$

Due to the Markov property of the solution, the family $\left(P_{s, t}\right)_{0 \leq s \leq t \leq T}$ fulfills the Chapman-Kolmogorov equation: $P_{s, t}=P_{r, t} \circ P_{s, r}$ for any $0 \leq s \leq r \leq t \leq T$.

Similarly to the Wiener noise case in BDPR09], the following result follows from Itô's formula. The proof uses a more concrete formulation of the operator $U$, which has been established in LR04, Prop. 3.5].

Lemma 4.2. For any $0 \leq s \leq t \leq T$, we have that $P_{s, t}\left(\mathcal{C}_{u}(H)\right) \subset \mathcal{C}_{u}(H)$. Furthermore, observe that for all $\psi \in \mathcal{W}_{T, A}$ we have

$$
P_{s, t} \psi(t, x)=\psi(s, x)+\int_{s}^{t} P_{s, r} L_{0} \psi(r, x) \mathrm{d} r \quad \text { for any } 0 \leq s \leq t \leq T \text { and } x \in H .
$$

4.1. Extension of the generator. Let $\alpha \in \mathbb{R}$ and $(s, x) \in[0, T] \times H$. Define

$$
R_{\alpha}^{L} \varphi(s, x):=\int_{s}^{T} e^{-\alpha(r-s)} \cdot P_{s, r} \varphi(r, \cdot)(x) \mathrm{d} r, \quad \varphi \in \mathcal{C}\left([0, T] ; \mathcal{C}_{u, 1}(H)\right) .
$$

Similarly to Section 3.2 we observe, that $R_{\alpha}^{L}$ is injective, $D\left(R_{\alpha}^{L}\right)=\mathcal{C}\left([0, T] ; \mathcal{C}_{u, 1}(H)\right)$, and that $R_{\alpha}^{L}\left(D\left(R_{\alpha}^{L}\right)\right)$ is independent of $\alpha$. We conclude that $\left(R_{\alpha}^{L}\right)^{-1}$ exists and is closed on $R_{\alpha}^{L}\left(D\left(R_{\alpha}^{L}\right)\right)$. Thus, $L:=\alpha I-\left(R_{\alpha}^{L}\right)^{-1}$ is also closed as a densely defined operator on $\mathcal{C}\left([0, T] ; \mathcal{C}_{u, 1}(H)\right)$. It is independent of $\alpha$, and

$$
R_{\alpha}^{L}=(\alpha I-L)^{-1} \quad \text { and } \quad D(L)=R_{\alpha}^{L}\left(\mathcal{C}\left([0, T] ; \mathcal{C}_{u, 1}(H)\right)\right) \quad \text { for all } \alpha \in \mathbb{R} .
$$

The space-time homogenization $P_{\tau}^{T}$ of $P_{s, t}$ in $\mathcal{C}_{T}\left([0, T] ; \mathcal{C}_{u, 1}(H)\right)$, given by

$$
\begin{array}{cl}
\left(P_{\tau}^{T} \varphi\right)(t, x) & \\
:= \begin{cases}P_{t, t+\tau} \varphi(t+\tau, \cdot)(x)=\mathbb{E}[\varphi(t+\tau, X(t+\tau, t, x))] & \text { for } t+\tau \leq T \\
0 & \text { otherwise, }\end{cases}
\end{array}
$$

is again a semigroup; similar to $\left(S_{\tau}^{T}\right)_{\tau \geq 0}$ before, $\left(P_{\tau}^{T}\right)_{\tau \geq 0}$ is a $\pi$-semigroup on $\mathcal{C}_{T}\left([0, T] ; \mathcal{C}_{u, 1}(H)\right)$. In the same way as in BDPR09, (2.10)], we can adapt Pri99 to have the following criterium to establish the generator $L$ of $P_{\tau}^{T}$ in the sense of $\pi$-semigroups and its domain $D(L)$ :

$$
\Leftrightarrow\left\{\begin{array}{c}
u \in D(L) \quad \text { and } \quad L u=\varphi \\
\lim _{h \rightarrow 0} \frac{1}{h}\left(\left(P_{h}^{T} u\right)(t, x)-u(t, x)\right)=\varphi(t, x) \quad \text { for all }(t, x) \in[0, T] \times H \\
\sup _{\substack{h \in(0,1],(t, x) \in[0, T] \times H}} \frac{(1+|x|)^{-1}}{h} \cdot\left|\left(P_{h}^{T} u\right)(t, x)-u(t, x)\right|<\infty .
\end{array}\right.
$$

To establish that $L$ extends $L_{0}$, we need to show that $\mathcal{W}_{T, A} \subset D(L)$ and that $L \psi=L_{0} \psi$ for all $\psi \in \mathcal{W}_{T, A}$. However, these facts both follow immediately from (4.1) together with Lemma 4.2 and an argument similar to the proof of Lemma 3.8 
In particular, for any $\psi \in \mathcal{W}_{T, A}, t<T$ and $t+h \leq T$, we have that there exists a $C \in(0, \infty)$ such that

$$
\begin{gathered}
\left(P_{h}^{T} \psi\right)(t, x)-\psi(t, x)=P_{t, t+h} \psi(t+h, \cdot)(x)-\psi(t, x)=\int_{t}^{t+h} P_{t, r} L_{0} \psi(r, x) \mathrm{d} r \\
\leq h \cdot C(1+|x|),
\end{gathered}
$$

where we use the definition of $L_{0}$, and the Lipschitz property of both $F$ (in space) and $X$ (with respect to the initial condition).

Lemma 4.3. Let $f \in \mathcal{C}\left([0, T] ; \mathcal{C}_{u}^{1}(H)\right)$ and $\alpha \in \mathbb{R}$. Set $u:=(\alpha I-L)^{-1} f$. Then,

(i) $D u \in \mathcal{C}\left([0, T] ; \mathcal{C}_{b}(H ; H)\right)$

(ii) $u \in D(V)$, and

$$
\alpha u-V u-\langle D u, F\rangle=f .
$$

In particular, $L u=V u+\langle D u, F\rangle$.

Using results from [MPR10], the proof of this lemma remains the same as in the Wiener noise case (see [BDPR09, Lem. 2.5]).

As mentioned in Remark 2.6, similar to the case of (SPDE) with regular drift term $F$ (cf. (H.6) driven by Wiener noise, $L$ can be shown to be m-dissipative.

For the next section, we need one more observation (which has been made in BDPR09, Rem. 1.1] for the case of (SPDE) driven by Wiener noise).

Remark 4.4. Let $\zeta \in \mathcal{M}_{1}(H)$ and $s \in[0, T]$. We note the following:

(i) Independent of the (non)regularity of $F$, any $\eta \in \mathcal{K}_{s, \zeta}^{0}$ fulfills for all $\psi \in$ $\mathcal{W}_{T, A}$

$$
\int_{s}^{T} \int_{H} L_{0} \psi(r, x) \eta_{r}(\mathrm{~d} x) \mathrm{d} r \leq 0 \quad \text { for all } \psi \in \mathcal{W}_{T, A} \text { with } \psi \geq 0 .
$$

(ii) If $\psi \in \mathcal{W}_{T, A}$, then $\psi^{2} \in \mathcal{W}_{T, A}$ (due to $\mathcal{W}_{T, A}$ being a sub-algebra of $\left.\mathcal{C}_{u}([0, T] \times H)\right)$, and the square field operator $\Gamma$ takes the form

$$
\begin{gathered}
\Gamma(\psi, \psi)(t, x):=L_{0} \psi^{2}(t, x)-2 \psi(t, x) \cdot L_{0} \psi(t, x) \\
=\langle D \psi(t, x), Q(D \psi(t, x))\rangle+\int_{H}(\psi(t, x)-\psi(t, x+y))^{2} M(\mathrm{~d} y),
\end{gathered}
$$

where $Q$ and $M$ are as in (1.1). Note, that both summands on the right hand side are nonnegative.

Using [LR04, Prop. 4.1], the observations of this remark follow from some simple computations.

\section{Measurable DRift}

In this section, we prove uniqueness of the solution to $(\mathrm{FPE})$ in the case of (SPDE) with a merely measurable nonlinear drift part $F$. Throughout this section, we assume that conditions (H.1) (H.5) hold. 
A main ingredient to the proof of this uniqueness result is a gradient estimate using the square-field operator $\Gamma$ introduced in Remark 4.4(ii) above. It is this estimate, which requires us to assume that $Q^{-1} \in L(H)$. Using this, we can adapt the approach of [BDPR11, Sect. 4], to obtain uniqueness of the solution to (FPE).

5.1. The dense range condition. We need to establish, that for $s \in[0, T]$ the dense range condition

$$
L_{0}\left(D\left(L_{0}\right)\right) \text { is dense in } L^{1}([s, T] \times H ; \eta)
$$

is fulfilled for any $\eta$ in a convex set $\mathcal{K}_{s, \leq 2 \alpha}^{\text {meas }}$ of measures to be defined below.

Definition 5.1. For $\alpha \geq 0$, we set

$\mathcal{K}_{s, \leq 2 \alpha}^{\text {meas }}:=\left\{\left.\eta \in \mathcal{K}_{s, \leq 2 \alpha}^{0}\left|\int_{[s, T] \times H}\right| x\right|^{2}+|F(t, x)|^{2}+|x|^{2} \cdot|F(t, x)|^{2} \eta(\mathrm{d} t, \mathrm{~d} x)<\infty\right\}$.

Lemma 5.2. Let $\alpha \geq 0, s \in[0, T]$ and $\eta \in \mathcal{K}_{s, \leq 2 \alpha}^{0}$. Then, for all $\psi \in \mathcal{W}_{T, A}$,

$$
\begin{gathered}
\int_{[s, T] \times H} \psi(t, x) L_{0} \psi(t, x) \eta(\mathrm{d} t, \mathrm{~d} x) \\
\leq \alpha \int_{[s, T] \times H} \psi^{2}(t, x) \eta(\mathrm{d} t, \mathrm{~d} x)-\frac{1}{2} \int_{[s, T] \times H} \Gamma(\psi, \psi)(t, x) \eta(\mathrm{d} t, \mathrm{~d} x) .
\end{gathered}
$$

In particular, $\left(L_{0}, \mathcal{W}_{T, A}\right)$ is quasi-dissipative, hence closable in $L^{2}([0, T] \times H, \eta)$ for any $\eta \in \mathcal{K}_{s, \leq 2 \alpha}^{\text {meas }}$; we denote the closure by $\left(L_{2}, D\left(L_{2}\right)\right)$.

Proof. By Remark 4.4(ii) and since $\mathcal{W}_{T, A}$ is a sub-algebra of $\mathcal{C}_{u}([0, T] \times H)=$ $\mathcal{C}\left([0, T] ; \mathcal{C}_{u}(H)\right)$, for any $\psi \in \mathcal{W}_{T, A}$ we have $\psi^{2} \in \mathcal{W}_{T, A}$ and

$$
\begin{gathered}
\int_{[s, T] \times H} \psi(t, x) \cdot L_{0} \psi(t, x) \eta(\mathrm{d} t, \mathrm{~d} x) \\
=\frac{1}{2} \int_{[s, T] \times H} L_{0} \psi^{2}(t, x) \eta(\mathrm{d} t, \mathrm{~d} x)-\frac{1}{2} \int_{[s, T] \times H} \Gamma(\psi, \psi)(t, x) \eta(\mathrm{d} t, \mathrm{~d} x),
\end{gathered}
$$

which proves the claim using (1.5).

Lemma 5.3. Let $\alpha>0, s \in[0, T], \eta \in \mathcal{K}_{s, \leq 2 \alpha}^{\text {meas }}$ and $f \in \mathcal{C}\left([s, T] ; \mathcal{C}_{u}^{1}(H)\right)$. Let $F_{c}$ : $[0, T] \times H \rightarrow H$ fulfill Hypothesis (H.6) and assume, that it fulfills the integrability condition in the definition of $\mathcal{K}_{s, \leq 2 \alpha}^{\text {meas }}$ above (together with $\eta$ ). Then, by Lemma 4.3. there is a $u_{c} \in D(V)$ with

$$
\alpha u_{c}-V u_{c}-\left\langle D u_{c}, F_{c}\right\rangle=f .
$$

By the m-dissipativity of $L$ in the case, where (H.6) is fulfilled, we obtain that $\left\|u_{c}\right\|_{0, T} \leq \frac{1}{\alpha} \cdot\|f\|_{0, T}$. In this situation, the following assertions hold:

(i) $u_{c} \in D\left(L_{2}\right)$ and

$$
\alpha u_{c}-L_{2} u_{c}=f+\left\langle D u_{c}, F_{c}-F\right\rangle \quad \text { in } L^{2}([s, T] \times H ; \eta) .
$$


(ii) We have

$$
\begin{gathered}
\int_{[s, T] \times H}\left|D u_{c}(t, x)\right|^{2} \eta(\mathrm{d} t, \mathrm{~d} x) \\
\leq 4\left\|Q^{-1}\right\| \cdot \frac{1}{\alpha^{2}} \cdot\|f\|_{0, T}^{2} \\
\cdot\left(\alpha \cdot(T-s)+\left\|Q^{-1}\right\| \int_{[s, T] \times H}\left|F_{c}(t, x)-F(t, x)\right|^{2} \eta(\mathrm{d} t, \mathrm{~d} x)\right) .
\end{gathered}
$$

Note, that the right hand side is finite for any $f \in \mathcal{C}\left([s, T] ; \mathcal{C}_{u}^{1}(H)\right)$.

Proof. By Corollary 3.12 there exists a sequence $\left(\psi_{n}\right)_{n \in \mathbb{N}} \subset \mathcal{W}_{T, A}$, such that for a constant $C_{3} \in(0, \infty)$, independent of $t$ and $x$, we have

$$
\left|\psi_{n}(t, x)\right|+\left|D \psi_{n}(t, x)\right|+\left|V_{0} \psi_{n}(t, x)\right| \leq C_{3} \cdot(1+|x|)
$$

for all $(t, x) \in[0, T] \times H, n \in \mathbb{N}$, and

$$
\psi_{n} \rightarrow u_{c}, \quad\left\langle D \psi_{n}, h\right\rangle \rightarrow\left\langle D u_{c}, h\right\rangle, \quad V_{0} \psi_{n} \rightarrow V u_{c}
$$

converge for any $h \in H$ in measure $\eta$ as $n \rightarrow \infty$.

Thus,

$$
L_{0} \psi_{n}=V_{0} \psi_{n}+\left\langle D \psi_{n}, F\right\rangle \stackrel{n \rightarrow \infty}{\longrightarrow} V u_{c}+\left\langle D u_{c}, F\right\rangle
$$

converges in measure $\eta$, and $\left|L_{0} \psi_{n}(t, \cdot)(x)\right| \leq C_{3} \cdot(1+|x|) \cdot(1+|F(t, x)|)$. Since $\eta \in \mathcal{K}_{s, \leq 2 \alpha}^{\text {meas }}$, we get with Lebesgue's dominated convergence theorem, that

$$
L_{0} \psi_{n} \stackrel{n \rightarrow \infty}{\longrightarrow} V u_{c}+\left\langle D u_{c}, F\right\rangle \quad \text { converges in } L^{2}([s, T] \times H ; \eta)
$$

and, consequently, $u_{c} \in D\left(L_{2}\right)$.

To complete the proof of (i), we simply recall that, by construction of $L_{2}$ and by assumption, we have

$$
L_{2} u_{c}-V u_{c}=\left\langle D u_{c}, F\right\rangle \text { and } \alpha u_{c}-V u_{c}=f+\left\langle D u_{c}, F_{c}\right\rangle .
$$

Let us consider (ii). Since $\eta \in \mathcal{K}_{s, \leq 2 \alpha}^{\text {meas }}$, we obtain from a similar approximation as above, that 5.2 holds for $u_{c} \in D\left(L_{2}\right)$ replacing $\psi$. This implies, that

$$
\begin{gathered}
\frac{1}{2} \int_{[s, T] \times H} \Gamma\left(u_{c}, u_{c}\right)(t, x) \eta(\mathrm{d} t, \mathrm{~d} x) \\
\leq \alpha \int_{[s, T] \times H} u_{c}^{2}(t, x) \eta(\mathrm{d} t, \mathrm{~d} x)-\int_{[s, T] \times H} u_{c}(t, x) \cdot L_{2} u_{c}(t, x) \eta(\mathrm{d} t, \mathrm{~d} x) .
\end{gathered}
$$

On the other hand, if we multiply (5.3) by $u_{c}$, we have

$$
\alpha u_{c}^{2}-u_{c} \cdot L_{2} u_{c}=u_{c} \cdot f+u_{c} \cdot\left\langle D u_{c}, F_{c}-F\right\rangle \quad \text { in } L^{2}([s, T] \times H ; \eta) .
$$

By (5.4), (5.5) and the definition of the square field operator $\Gamma$ we conclude, that

$$
\begin{gathered}
\frac{1}{2} \int_{[s, T] \times H}\left|Q^{1 / 2}\left(D u_{c}(t, x)\right)\right|^{2} \eta(\mathrm{d} t, \mathrm{~d} x) \\
+\underbrace{\int_{[s, T] \times H} \int_{H}\left(u_{c}(t, x)-u_{c}(t, x+y)\right)^{2} M(\mathrm{~d} y) \eta(\mathrm{d} t, \mathrm{~d} x)}_{\geq 0}
\end{gathered}
$$




$$
\begin{gathered}
\leq \int_{[s, T] \times H}\left|u_{c}(t, x)\right| \cdot|f(t, x)| \eta(\mathrm{d} t, \mathrm{~d} x) \\
+\int_{[s, T] \times H}\left|u_{c}(t, x)\right| \cdot\left|D u_{c}(t, x)\right| \cdot\left|F_{c}(t, x)-F(t, x)\right| \eta(\mathrm{d} t, \mathrm{~d} x) \\
\leq(T-s) \cdot \frac{1}{\alpha} \cdot\|f\|_{0, T}^{2} \\
\quad+\int_{[s, T] \times H} \frac{1}{2} \cdot \frac{1}{2\left\|Q^{-1}\right\|} \cdot\left|D u_{c}(t, x)\right|^{2} \\
+\frac{1}{2} \cdot 2\left\|Q^{-1}\right\| \cdot \frac{1}{\alpha^{2}} \cdot\|f\|_{0, T}^{2} \cdot\left|F_{c}(t, x)-F(t, x)\right|^{2} \eta(\mathrm{d} t, \mathrm{~d} x)
\end{gathered}
$$

where we used Young's inequality in the last step. This implies the assertion of the Lemma.

Proposition 5.4. Let $\alpha>0, s \in[0, T]$ and $\eta \in \mathcal{K}_{s, \leq 2 \alpha}^{\text {meas }}$. Then, the dense range condition (5.1) is fulfilled.

Proof. For any measurable map $F: D(F) \subset[0, T] \times H \rightarrow H$ and $\eta \in \mathcal{K}_{s, \leq 2 \alpha}^{\text {meas }}$, there exists a sequence $\left(F_{n}\right)_{n \in \mathbb{N}}$ of functions, which fulfill Hypothesis (H.6)] and

$$
\lim _{n \rightarrow \infty} \int_{[s, T] \times H}\left|F_{n}-F\right|^{2} \mathrm{~d} \eta=0 .
$$

Let $f \in \mathcal{C}\left([s, T] ; \mathcal{C}_{u}^{1}(H)\right)$. Then, by Lemma 4.3 and Corollary 3.12 , there exists for any $n \in \mathbb{N}$ a function $\psi_{n} \in \mathcal{W}_{T, A}$, such that

$$
\alpha \psi_{n}-V \psi_{n}-\left\langle D \psi_{n}, F_{n}\right\rangle=f .
$$

By m-dissipativity of $L$ in the regular case, we have $\left\|\psi_{n}\right\|_{0, T} \leq \frac{1}{\alpha} \cdot\|f\|_{0, T}$. Thus, by Lemma 5.3 (i), for any $n \in \mathbb{N}$

$$
\alpha \psi_{n}-L_{2} \psi_{n}=f+\left\langle D \psi_{n}, F_{n}-F\right\rangle \quad\left(\text { in } L^{2}\right),
$$

and from Lemma 5.3(ii) we obtain that

$$
\sup _{n \in \mathbb{N}} \int_{[s, T] \times H}\left|D \psi_{n}(t, x)\right|^{2} \eta(\mathrm{d} t, \mathrm{~d} x)<\infty .
$$

Together, 5.6) (5.8) imply (as $n \rightarrow \infty)$, that $f$ is in the closure of $\left(\alpha-L_{0}\right)\left(\mathcal{W}_{T, A}\right)$ in $L^{1}([s, T] \times H ; \eta)$.

Now, since $f$ is arbitrarily chosen from $\mathcal{C}\left([s, T] ; \mathcal{C}_{u}^{1}(H)\right)$, and $\operatorname{since} \mathcal{C}\left([s, T] ; \mathcal{C}_{u}^{1}(H)\right)$ is dense in $L^{1}([s, T] \times H ; \eta)$, the dense range condition (5.1) is shown.

5.2. Uniqueness of the solution to the Fokker-Planck equation. We return to our initial aim, to establish a proof for Theorem 2 .

Since $\mathcal{K}_{s, \leq 2 \alpha}^{\text {meas }}$ is a superset of $\mathcal{K}_{s, \zeta}^{\text {meas }}$ (by virtue of $\mathcal{K}_{s, \leq 2 \alpha}^{0}$ being a superset of $\mathcal{K}_{s, \zeta}^{0}$; see Remark 4.4(i) and (4.2)), we know by the preceding subsection, that any $\eta \in \mathcal{K}_{s, \zeta}^{\text {meas }}$ fulfills the dense range condition (5.1). As a consequence, we get the uniqueness of the solution to (FPE) from the following result. The proof is a standard argument, which we include here only for the convenience of the reader.

Proposition 5.5. Let $\tilde{\mathcal{K}} \subset \mathcal{K}_{s, \zeta}^{0}$ be a convex subset, such that the dense range condition [5.1] is fulfilled for any $\eta \in \tilde{\mathcal{K}}$.

Then, $\mathcal{K}$ contains at most one element. 
Proof. Let $\eta^{(1)}, \eta^{(2)} \in \tilde{\mathcal{K}}$ and set $\mu:=\frac{1}{2} \eta^{(1)}+\frac{1}{2} \eta^{(2)}$. Then $\mu \in \tilde{\mathcal{K}}$, and $\eta^{(i)}=\sigma_{i} \mu$ for some measurable functions $\sigma_{i}:[s, T] \times H \rightarrow[0,2]$, for $i \in\{1,2\}$.

By 4.2 we see, that

$$
\int_{[s, T] \times H} L_{0} \psi \mathrm{d} \eta^{(1)}=\int_{[s, T] \times H} L_{0} \psi \mathrm{d} \eta^{(2)} \quad \text { for all } \psi \in \mathcal{W}_{T, A},
$$

in other words,

$$
\int_{[s, T] \times H} L_{0} \psi\left(\sigma_{1}-\sigma_{2}\right) d \mu=0 \quad \text { for all } \psi \in \mathcal{W}_{T, A} .
$$

However, by Proposition 5.4 the range of $\left(L_{0}, \mathcal{W}_{T, A}\right)$ is dense in $L^{1}([s, T] \times H ; \mu)$. Also, $\left(\sigma_{1}-\sigma_{2}\right)$ is bounded by definition. Thus, we see that $\sigma_{1}=\sigma_{2}$.

\section{EXAMPLE}

One classical example for semilinear equations of type (SPDE) are reactiondiffusion equations, where the linear part describes the diffusion of substances e.g. in a fluid, and the nonlinear part describes the space-time development of a reaction (e.g. chemical or biological) between these substances.

In this section, we explain the application of our results to such a situation. Let us note two things. First, it is not proven at this moment, in which way existing existence results for solutions to (FPE) from the Wiener noise case can be adapted to our framework. We thus have to assume for this section, that existence results similar to those from the Wiener noise case exist within our framework. Second, what follows below is naturally not the first description of how to apply abstract results for Fokker-Planck equations characterizing the solutions of SPDE to reaction-diffusion problems. Our presentation below is structured along the lines of that in BDPR11, Sect. 6].

Let $H:=L^{2}((0,1))$ (the $L^{2}$-space with respect to the Lebesgue measure on $(0,1) \subset \mathbb{R})$ with norm $|\cdot|_{H}=|\cdot|_{L^{2}((0,1))}$. Define the linear operator $A: D(A) \subset$ $H \rightarrow H$ by $A x(r):=\frac{\mathrm{d}^{2}}{\mathrm{dr}^{2}} x(r)$ and $D(A):=H^{2}((0,1)) \cap H_{0}^{1}((0,1))$. This operator fulfills Hypothesis (H.1)

The nonlinear drift part $F: D(F) \rightarrow H$ is defined, for a given $m \in \mathbb{N}$, on $D(F):=[0, T] \times L^{2 m}((0,1))$ by

$$
F(t, x)(r):=f(r, t, x(r))+h(r, t, x(r)) \quad \text { for all } r \in(0,1),(t, x) \in D(F),
$$

where we assume that $f, h:(0,1) \times[0, T] \times \mathbb{R} \rightarrow \mathbb{R}$ are measurable functions, which fulfill the following conditions:

(0) For any fixed $r \in(0,1)$, the functions $f(r, \cdot, \cdot)$ and $h(r, \cdot, \cdot)$ are continuous on $[0, T] \times \mathbb{R}$.

(f1) (polynomial growth). There exist an odd integer $m \in \mathbb{N}$ and a nonnegative $c_{1} \in L^{2}([0, T])$, such that

$|f(r, t, z)|_{H} \leq c_{1}(t) \cdot\left(1+|z|_{H}^{m}\right) \quad$ for all $t \in[0, T], z \in \mathbb{R}, r \in(0,1)$.

(f2) (quasi-dissipativity). There exists a nonnegative $c_{2} \in L^{1}([0, T])$, such that

$$
\begin{aligned}
& {\left[f\left(r, t, z_{1}\right)-f\left(r, t, z_{2}\right)\right] \cdot\left(z_{1}-z_{2}\right) \leq c_{2}(t) \cdot\left|z_{1}-z_{2}\right|_{H}^{2}} \\
& \text { for all } t \in[0, T] ; z_{1}, z_{2} \in \mathbb{R} ; r \in(0,1) .
\end{aligned}
$$


(h1) (linear growth). There exists a nonnegative $c_{3} \in L^{2}([0, T])$, such that

$$
|h(r, t, z)|_{H} \leq c_{3}(t) \cdot\left(1+|z|_{H}\right) \quad \text { for all } t \in[0, T], z \in \mathbb{R}, r \in(0,1) .
$$

For such a nonlinear drift part, it is not yet established, whether there exists a pathwise solution to equations of type (SPDE) (for either Wiener or more general noise). However, $F$ as described above fits into the framework of Section 5 , fulfilling (H.4)

Assume, that Hypotheses (H.2) (H.3) and (H.5) are fulfilled. Set $c_{4}(t):=$ $2\left[c_{1}(t)+c_{3}(t)+1\right]$ and define, for $N \in \mathbb{N}$,

$$
V_{N}(t, x):= \begin{cases}c_{4}(t) \cdot\left(1+|x|_{L^{2 N}((0,1))}^{N}\right) & \text { if }(t, x) \in[0, T] \times L^{2 N}((0,1)) \\ +\infty & \text { else. }\end{cases}
$$

Observe, that (f1) and (h1) imply

$$
|F(t, x)|_{H} \leq V_{m}(t, x)<\infty .
$$

Assume that from here on $N \geq m$.

Assumption 6.1. For any $\zeta \in \mathcal{M}_{1}(H)$ with $\int_{H}|x|_{L^{2 N}((0,1))}^{2 N} \zeta(\mathrm{d} x)<\infty$, there exists a solution $\eta \in \mathcal{K}_{s, \zeta}^{0}$ to (FPE.

This solution has the following additional properties:

$$
\begin{gathered}
\sup _{t \in[s, T]} \int_{H}|x|_{H}^{2} \eta_{t}(\mathrm{~d} x)<\infty \\
t \mapsto \int_{H} \psi(t, x) \eta_{t}(\mathrm{~d} x) \quad \text { is continuous for all } \psi \in \mathcal{W}_{T, A}
\end{gathered}
$$

$$
\leq C_{5} \cdot \int_{s}^{T} \int_{H} V_{N}^{2}(r, x) \zeta(\mathrm{d} x) \mathrm{d} r<\infty \quad \text { for } a C_{5} \in(0, \infty) \text { and any } \delta \in\left(0, \frac{1}{2}\right) \text {. }
$$

(Note, that any Dirac measure $\delta_{x}$ with $x \in L^{2 N}((0,1))$ fulfills the requirements for $\zeta$ indicated above.)

Remark 6.2. This Assumption is proven to be valid for the Wiener noise case in BDPR10, Sect. 4]. The approach from the Wiener noise case, involving a technique of subtracting the noise in combination with an application of Itô's formula, can be generalized. The details, that this will lead to an existence proof for the case of noise with jumps, will be the topic of a forthcoming paper.

Choose now $N:=m+2$ and observe that by construction of $V_{N}$ and 6.1 (note that $c_{4}(t) \geq 1$ for all $t$ by definition),

$$
\begin{gathered}
\int_{s}^{T} \int_{H}|F(t, x)|_{H}^{2} \eta_{t}(\mathrm{~d} x) \mathrm{d} t \leq \int_{s}^{T} \int_{H} V_{m}^{2}(t, x) \eta_{t}(\mathrm{~d} x) \mathrm{d} t \\
\leq \int_{s}^{T} \int_{H}\left[c_{4}(t) \cdot\left(1+|x|_{L^{2 m}((0,1))}^{m}\right]^{2} \eta_{t}(\mathrm{~d} x) \mathrm{d} t\right. \\
\leq \int_{s}^{T} c_{4}^{2}(t) \int_{H} 1+|x|_{L^{2 m}((0,1))}^{2 m} \eta_{t}(\mathrm{~d} x) \mathrm{d} t
\end{gathered}
$$


which implies, together with the properties of our assumedly existing solution $\eta$, that $\eta$ is in $\mathcal{K}_{s, \zeta}^{\text {meas }}$, if the initial condition $\zeta$ fulfills

$$
\int_{H}|x|_{L^{2(m+2)}((0,1))}^{2(m+2)} \zeta(\mathrm{d} x)<\infty .
$$

Consequently, by Theorem 2, $\eta$ is the only solution to FPE with coefficients and initial data as specified above.

\section{REFERENCES}

[AF09] L. Ambrosio and A. Figalli, On flows associated to Sobolev vector fields in Wiener spaces: an approach à la DiPerna-Lions, J. Funct. Anal. 256 (2009), no. 1, 179-214, DOI 10.1016/j.jfa.2008.05.007.

[App07] D. Applebaum, On the infinitesimal generators of Ornstein-Uhlenbeck processes with jumps in Hilbert space, Potential Anal. 26 (2007), no. 1, 79-100, DOI 10.1007/s11118006-9028-y.

[BDPR08] V.I. Bogachev, G. Da Prato, and M. Röckner, On parabolic equations for measures, Comm. Partial Differential Equations 33 (2008), no. 1-3, 397-418, DOI 10.1080/03605300701382415.

[BDPR09] _ Fokker-Planck equations and maximal dissipativity for Kolmogorov operators with time dependent singular drifts in Hilbert spaces, J. Funct. Anal. 256 (2009), no. 4, 1269-1298, DOI 10.1016/j.jfa.2008.05.005.

[BDPR10] _ Existence and uniqueness of solutions for Fokker-Planck equations on Hilbert spaces, J. Evol. Equ. 10 (2010), no. 3, 487-509, DOI 10.1007/s00028-010-0058-y.

[BDPR11] _ Uniqueness for solutions of Fokker-Planck equations on infinite dimensional spaces, Communications in Partial Differential Equations 36 (2011), 925-939.

[BLR99] V.I. Bogachev, P. Lescot, and M. Röckner, The martingale problem for pseudodifferential operators on infinite-dimensional spaces, Nagoya Math. J. 153 (1999), 101-118.

[BRS96] V.I. Bogachev, M. Röckner, and B. Schmuland, Generalized Mehler semigroups and applications, Probab. Theory Related Fields 105 (1996), no. 2, 193-225, DOI 10.1007/BF01203835.

[CM87] A. Chojnowska-Michalik, On processes of Ornstein-Uhlenbeck type in Hilbert space, Stochastics 21 (1987), no. 3, 251-286.

[Cer95] S. Cerrai, Weakly continuous semigroups in the space of functions with polynomial growth, Dynam. Systems Appl. 4 (1995), no. 3, 351-371.

[DP04] G. Da Prato, Kolmogorov equations for stochastic PDEs, Advanced Courses in Mathematics. CRM Barcelona, Birkhäuser Verlag, Basel, 2004.

[DP06] _ An introduction to infinite-dimensional analysis, Universitext, SpringerVerlag, Berlin, 2006.

[DPT01] G. Da Prato and L. Tubaro, Some results about dissipativity of Kolmogorov operators, Czechoslovak Math. J. 51(126) (2001), no. 4, 685-699, DOI 10.1023/A:1013704610695.

[DPZ92] G. Da Prato and J. Zabczyk, Stochastic equations in infinite dimensions, Encyclopedia of Mathematics and its Applications, vol. 44, Cambridge University Press, Cambridge, 1992.

[DPZ02] _ Second order partial differential equations in Hilbert spaces, London Mathematical Society Lecture Note Series, vol. 293, Cambridge University Press, Cambridge, 2002.

[DL89] R.J. DiPerna and P.-L. Lions, Ordinary differential equations, transport theory and Sobolev spaces, Invent. Math. 98 (1989), no. 3, 511-547, DOI 10.1007/BF01393835.

[Fig08] A. Figalli, Existence and uniqueness of martingale solutions for SDEs with rough or degenerate coefficients, J. Funct. Anal. 254 (2008), no. 1, 109-153, DOI 10.1016/j.jfa.2007.09.020.

[FR00] M. Fuhrman and M. Röckner, Generalized Mehler semigroups: the non-Gaussian case, Potential Anal. 12 (2000), no. 1, 1-47, DOI 10.1023/A:1008644017078.

[Hai09] M. Hairer, An Introduction to Stochastic PDEs, 2009. Lecture Notes, available through the author's website. 
[LBL08] C. Le Bris and P.-L. Lions, Existence and uniqueness of solutions to Fokker-Planck type equations with irregular coefficients, Comm. Partial Differential Equations 33 (2008), no. 7-9, 1272-1317, DOI 10.1080/03605300801970952.

[LR02] P. Lescot and M. Röckner, Generators of Mehler-type semigroups as pseudo-differential operators, Infin. Dimens. Anal. Quantum Probab. Relat. Top. 5 (2002), no. 3, 297-315, DOI 10.1142/S0219025702000894.

[LR04] , Perturbations of generalized Mehler semigroups and applications to stochastic heat equations with Levy noise and singular drift, Potential Anal. 20 (2004), no. 4, 317-344, DOI 10.1023/B:POTA.0000009814.54278.34.

[Lin86] W. Linde, Probability in Banach spaces - stable and infinitely divisible distributions, 2nd ed., A Wiley-Interscience Publication, John Wiley \& Sons Ltd., Chichester, 1986.

[MR92] Z.-M. Ma and M. Röckner, Introduction to the theory of (nonsymmetric) Dirichlet forms, Universitext, Springer-Verlag, Berlin, 1992.

[Man06] L. Manca, Kolmogorov operators in spaces of continuous functions and equations for measures, Dissertation, Scuola Normale Superiore di Pisa, 2006.

[MPR10] C. Marinelli, C.I. Prévôt, and Michael Röckner, Regular dependence on initial data for stochastic evolution equations with multiplicative Poisson noise, J. Funct. Anal. 258 (2010), no. 2, 616-649, DOI 10.1016/j.jfa.2009.04.015.

[MR10] C. Marinelli and M. Röckner, Well-posedness and asymptotic behavior for stochastic reaction-diffusion equations with multiplicative Poisson noise, Electron. J. Probab. 15 (2010), no. 49, 1528-1555.

[Par67] K.R. Parthasarathy, Probability measures on metric spaces, Probability and Mathematical Statistics, No. 3, Academic Press Inc., New York, 1967.

[PZ07] S. Peszat and J. Zabczyk, Stochastic partial differential equations with Lévy noise, Encyclopedia of Mathematics and its Applications, vol. 113, Cambridge University Press, Cambridge, 2007.

[Pri99] E. Priola, On a class of Markov type semigroups in spaces of uniformly continuous and bounded functions, Studia Math. 136 (1999), no. 3, 271-295.

[RS80] M. Reed and B. Simon, Methods of modern mathematical physics. I, 2nd ed., Academic Press Inc. [Harcourt Brace Jovanovich Publishers], New York, 1980. Functional analysis.

[RZ10] M. Röckner and X. Zhang, Weak uniqueness of Fokker-Planck equations with degenerate and bounded coefficients, C. R. Math. Acad. Sci. Paris 348 (2010), no. 7-8, 435-438, DOI 10.1016/j.crma.2010.01.001.

[SS01] B. Schmuland and W. Sun, On the equation $\mu_{t+s}=\mu_{s} * T_{s} \mu_{t}$, Statist. Probab. Lett. 52 (2001), no. 2, 183-188, DOI 10.1016/S0167-7152(00)00235-2.

[Wie11] S. Wiesinger, Uniqueness of solutions to Fokker-Planck equations related to singular SPDE driven by Lévy and cylindrical Wiener noise, Dissertation, Bielefeld University, 2011, http://www.math.uni-bielefeld.de/ bibos/preprints/E12-01-395.pdf.

Department of Mathematics, Bielefeld University, Bielefeld, Germany

E-mail address: swiesing@math.uni-bielefeld.de 